\title{
Jumps in option prices and their determinants: Real-time evidence from the E-mini S\&P 500 options market
}

DOI:

10.1016/j.finmar.2019.100506

\section{Document Version}

Accepted author manuscript

Link to publication record in Manchester Research Explorer

\section{Citation for published version (APA):}

Kapetanios, G., Konstantinidi, E., Skiadopoulos, G., \& Neumann, M. (2019). Jumps in option prices and their determinants: Real-time evidence from the E-mini S\&P 500 options market. Journal of Financial Markets, 46(0), [100506]. https://doi.org/10.1016/j.finmar.2019.100506

\section{Published in:}

Journal of Financial Markets

\section{Citing this paper}

Please note that where the full-text provided on Manchester Research Explorer is the Author Accepted Manuscript or Proof version this may differ from the final Published version. If citing, it is advised that you check and use the publisher's definitive version.

\section{General rights}

Copyright and moral rights for the publications made accessible in the Research Explorer are retained by the authors and/or other copyright owners and it is a condition of accessing publications that users recognise and abide by the legal requirements associated with these rights.

\section{Takedown policy}

If you believe that this document breaches copyright please refer to the University of Manchester's Takedown Procedures [http://man.ac.uk/04Y6Bo] or contact uml.scholarlycommunications@manchester.ac.uk providing relevant details, so we can investigate your claim.

\section{OPEN ACCESS}




\title{
Jumps in option prices and their determinants: Real-time evidence from the E-mini S\&P 500 option market $\$$
}

\author{
George Kapetanios, ${ }^{a}$ Eirini Konstantinidi, \\ Michael Neumann, George Skiadopoulos ${ }^{d}$
}

July 24, 2019

\begin{abstract}
We provide first-time evidence of the real-time characteristics and drivers of jumps in option prices. To this end, we employ high frequency data from the 24-hour E-mini S\&P 500 options market. We find that option prices do not jump simultaneously across strikes and maturities and are uncorrelated with jumps in the underlying futures price. $14 \%$ to $28 \%$ of detected option price jumps occur around scheduled news releases. However, it is illiquidity rather than the news content that drives jumps. Evidence suggests that option traders increase bid-ask spreads to account for trading against investors who are skilled processors of public releases. Interestingly, illiquidity does not drive jumps in the highly liquid S\&P 500 index options market, where we also find sizable and idiosyncratic price jumps.
\end{abstract}

Keywords: Asymmetric information, Co-jump, Limit order book market, Liquidity, Option Market, News announcement

JEL Classification: C58, G10, G12, G13

\footnotetext{
$\S$ We would like to thank Richard Baillie, Alejandro Bernales, Menachem Brenner, Francis Breedon, Marcelo Fernandes, Robert Geske, Arie Gozluklu, Massimo Guidolin, Michel Habib, Erik Hjalmarsson, Andrew Karolyi, Arie Melnik, Dmitryi Muravyev, Anthony Neuberger, Neil Pearson, Tatjana Puhan, Olivier Scaillet, Ben Schreiber, Spyros Skouras, Theodoros Stamatiou, Zvi Wiener, Gal Zahavi, and participants at the Bank of England, Luxembourg School of Finance, University of Piraeus, University of Zurich seminar series, the 2013 MMF Workshop on Empirical Modelling of Financial Markets at Brunel University, the 2014 Jerusalem Finance Conference in honour of Professors Dan Galai and Itzhak Venezia at Hebrew University, the 2014 European Financial Management Conference (Rome), the 2014 Conference on Extreme Events in Finance at ESSEC Business School, the 2015 Conference on Recent Advances in Finance at Queen Mary University of London, and the 2018 Conference on Research on Economic Theory and Econometrics for useful discussions and comments. Financial support from the Chicago Mercantile Exchange Group Foundation and the University of Piraeus Research Centre of is gratefully acknowledged. All remaining errors are solely the authors' responsibility.

${ }^{a}$ King's Business School, King's College London, UK, george.kapetanios@kcl.ac.uk

${ }^{b}$ Alliance Manchester Business School, University of Manchester, UK, eirini.konstantinidi@mbs.ac.uk

${ }^{c}$ Independent, michael.neumann.ln@gmail.com

${ }^{d}$ Corresponding author. School of Economics and Finance, Queen Mary, University of London, UK, and Department of Banking and Financial Management, University of Piraeus, Greece. Also Associate Research Fellow with Cass Business School and Warwick Business School, g.skiadopoulos@qmul.ac.uk, gskiado@unipi.gr
} 


\section{Introduction}

We provide first-time evidence on the characteristics and drivers of discontinuous changes, termed jumps, in option prices. We address four questions: Do jumps in option prices occur (1) simultaneously across strikes and maturities?, (2) as a result of jumps in the underlying asset market?, (3) as a result of news announcements?, and (4) as a result of shrinkages in liquidity?

These research questions are motivated by financial theory and they are of importance to both academics and practitioners for three reasons. First, options have emerged as an important asset class and a number of studies examine their risk-return profile (Coval and Shumway, 2001; Driessen and Maenhout, 2007; Broadie et al., 2009; Santa-Clara and Saretto, 2009). Second, any option pricing model should generate the empirical characteristics of jumps to be consistent with the data. This is of particular importance in the context of option pricing models built to be consistent with the dynamics of market option prices (Jackwerth, 1999; Skiadopoulos, 2001). Third, the identification of option jumps characteristics and determinants can shed light on the way that option prices are being formed in real-time.

To study the fine structure and real-time determinants of jumps, we employ high frequency option quotes on the S\&P 500 E-mini futures options trading in a nearly 24-hour limit order book electronic market at the Chicago Mercantile Exchange (CME). We classify traded option contracts in eighteen strike and time-to-maturity categories and we compute 10-minutes option returns for any given strike and maturity bucket. Then, we identify price jumps and their exact timings using Lee and Mykland's (2008) (LM, thereafter) jump detection test.

Next, we investigate the nature of detected option price jumps and their relation with three classes of determinants. First, we study whether option price jumps stem from jumps in the underlying asset's price and/or its volatility. Option pricing theory states that the dynam- 
ics of option prices are dictated by the dynamics of the price and volatility of the underlying asset. Second, we examine whether the occurrence as well as the content of news releases is associated with jumps. The release of news is expected to trigger jumps in option prices via two channels: heterogeneous beliefs (Shefrin, 2001; Buraschi and Jiltsov, 2006; Friesen et al., 2012) and market sentiment (Han, 2008; Lemmon and Ni, 2011). We employ a set of U.S. scheduled macroeconomic news announcements which are well monitored by academics and practitioners. ${ }^{1}$ The investigation of the real-time relation between jumps and scheduled news announcements is possible because our 24-hour dataset includes the times at which most scheduled U.S. macroeconomic news announcements are released. We also employ a comprehensive list of unscheduled news announcements; existing studies have paid little attention to the effects of unscheduled news to asset prices. Third, we investigate whether the detected jumps in option prices may be due to changes in the liquidity of the option market. Christoffersen et al. (2018) find that option illiquidity predicts future option price increases.

We find that option prices jump. The probability of a jump to occur ranges from $0.22 \%$ to $0.56 \%$ depending on the option strike and maturity. Jumps are found to be negative on average, they are sizeable with an average size up to $63 \%$ of the option price and they are mostly idiosyncratic, i.e. option prices in one strike and maturity category tend to jump independently from prices in other categories. This implies that the options market does not behave homogeneously in terms of the discontinuous movements of its prices. This finding is not be surprising given that the $\mathrm{S} \& \mathrm{P} 500 \mathrm{E}$-mini futures options is populated by traders with different motives and it extends Sheikh and Ronn (1994) who provide evidence on the heterogeneity of the put and call option raw returns rather than the jump price dynamics trading in a limit order book market, too.

\footnotetext{
${ }^{1}$ We do not employ firm specific announcements because the underlying asset in the employed options has to do with the aggregate market. The aggregate market will be affected by firm-specific news only to the extent that a firm has a dominant position in the market; there is no reason to expect that this is the case.
} 
We find that option price jumps are mostly unrelated to jumps in the underlying asset's price. These results complement the findings of Bakshi et al. (2000) who document that index call (put) prices do not always move in the same (opposite) direction with the underlying index and their dynamics differ across strikes and maturities. We document that $14 \%$ to $28 \%$ of the identified jumps occur around scheduled macroeconomic news releases depending on the strike and maturity. However, even though a fraction of jumps clusters around news announcements, we find that market illiquidity rather than the news content drives jumps in option prices. We also find that jumps unrelated to the release of scheduled news are also triggered by shrinking market liquidity. The shrinkage of market liquidity is manifested by an increase in options bid-ask spreads at the jump occurrence. These results are robust to the choice of the sample period (non-crisis versus crisis periods) and to the choice of the set of news releases (scheduled versus unscheduled news items).

Our findings on the relation between market liquidity and news-related jumps are consistent with the existence of informed trading in option markets (Chan et al., 1995; Easley et al., 1998; Chakravarty et al., 2004; Pan and Poteshman, 2006, and references therein). ${ }^{2}$ Option traders quote wider bid-ask spreads and thus they decrease market liquidity just before the macroeconomic news announcement to avoid trading with informed agents. This is consistent with Handa et al. (2003) who show that bid-ask spreads are a function of information asymmetry in a limit order book market. Moreover, our results extend the evidence by Erenburg and Lasser (2009) who find that in a limit order book market, the index-linked securities bid-ask spreads increase around macroeconomic news releases.

We document that most of the news-related jumps are accompanied by zero trading vol-

\footnotetext{
${ }^{2}$ In the case of a dealers market, an increase in the option's bid-ask spread can also be attributed to the increase in inventory costs (Muravyev, 2016) and /or to the hedging costs of option market makers (Huh et al., 2014). However, the option market under consideration is not a dealers market since quotes can be provided by any type of investor; no information can be obtained on the type of investor who places orders.
} 
ume. This has two important implications. First, there are no informed option traders prior to scheduled macroeconomic announcements in the sense that there is no leakage of private information; if it were, then trading activity should take place prior to the announcement. This is inline with Ederington and Lee (1995) who find that there is no information leakage prior to scheduled news releases in the context of interest rate and foreign currency futures markets. Interestingly, this finding is in contrast to the evidence that there is private information prior to company specific announcements (Augustin et al., 2019 and references therein). Second, our finding on the relation between jumps and volume sheds light on the type of information asymmetry that traders are concerned about in option markets. Kim and Verrecchia (1994) and Kim and Verrecchia (1997) define two types of private information: private information which accrues to some investors due to leakage of information prior to an announcement and information which accrues to investors who are skilled in processing publicly announced information and thus effectively converting it to private. This is consistent with Kandel and Pearson (1995) who provide evidence that stock market participants interpret the same news release differently. Our findings suggest that option traders increase bid-ask spreads because they may also interact with investors who possess the latter type of private information.

We extend our analysis by considering S\&P 500 index options which are actively traded on the Chicago Board of Exchange (CBOE). We find that jumps are idiosyncratic just as it was the case in the E-mini options market. We also document that a fraction of the detected jumps is linked to scheduled macroeconomic news announcements; in $6 \%$ to $27 \%$ of the cases a news announcement triggers a jump. Finally, we find that the option market illiquidity is not the key determinant of option jumps in this market.

We conclude this introduction by discussing four related strands of literature that our paper also contributes to. First, a number of studies find that a portion of jumps in asset prices are 
related to news announcements in the context of equities (Maheu and McCurdy, 2004; Rangel, 2011; Evans, 2011), bonds (Jiang et al., 2011), stock index futures, bond futures and exchange rates (Lahaye et al., 2011). Jiang et al. (2011) and Boudt and Petitjean (2014) also find that changes in liquidity result in jumps in bond and equity prices, respectively.

Second, there is an extensive literature which investigates the real-time option price formation (Vijh, 1990; George and Longstaff, 1993; Sheikh and Ronn, 1994; Chan et al., 1995; Berkman, 1996; Chan et al., 2002; Chakravarty et al., 2004; Muravyev, 2016, among others). However, this literature does not distinguish between continuous and discontinuous option price movements and it also considers equity options. To the best of our knowledge, Taylor et al. (2013) is the only other study which explores the presence of jumps in option markexts using high-frequency option prices; they consider options written on FTSE 100. However, their scope differs from ours. Taylor et al. (2013) investigate which option pricing model can generate the detected option jumps, whereas we explore their economic sources.

Third, various studies examine the time evolution of the S\&P 500 implied volatilities (Skiadopoulos et al., 1999; Gonçalves and Guidolin, 2006; Neumann and Skiadopoulos, 2013). Again, these studies do not identify whether the observed changes in implied volatilities are smooth or discontinuous. Finally, previous studies explore the effect of news announcements on at-the-money equity options implied volatilities (Ederington and Lee, 1996; Fornari and Mele, 2001) as well as the option-implied VIX (Bailey et al., 2014). However, these papers do not investigate whether the impact of news releases creates discontinuities in option prices and they do not examine the entire spectrum of traded options individually. Most importantly, they explore the impact of news releases whereas we take the reverse approach by detecting first jumps and then we check their sources in the vicinity of their occurrence.

The remainder of this paper is organized as follows. Section 2 describes the E-mini S\&P 
500 dataset and the way we structure it for the purposes of our analysis. Section 3 introduces and applies Lee and Mykland's (2008) test to identify jumps in option prices across different strike and maturity categories. Section 4 investigates the determinants of option price jumps. Section 5 conducts a number of robustness checks and Section 6 considers the case of S\&P 500 index options. Section 7 concludes and outlines the implications of our research.

\section{Data}

\subsection{Option data}

We obtain intra-day data for S\&P 500 E-mini futures options and the underlying futures (E-mini hereafter) from CME DataMine spanning 1 January 2005 to 31 December 2010. The dataset includes the best bid and ask quotes time-stamped down to the second, the sizes quoted at the best bid and ask prices, the trading volume and transaction prices. Both options and futures contracts trade in a nearly 24-hour electronic market termed GLOBEX. ${ }^{3}$ The use of this dataset is of utmost importance for the purposes of our study because in the subsequent analysis it will allow us to identify any real-time association of detected jumps with the scheduled U.S. news releases. This is because most scheduled U.S. news announcements are released at 7:30 a.m. Central Standard Time (CST) taking place outside of the trading hours of most organized equity derivative exchanges. However, a real-time analysis is required as news announcement effects have been found to be relatively short-lived (for a similar choice in the context of futures markets, see e.g., Andersen et al., 2007). We sample quotes from 7.00 a.m. to 2.45 p.m. CST

\footnotetext{
3 "E-mini" contracts are sized at one-fifth of the value of the regular contracts, making them more accessible to traders with small margin accounts. They trade almost continuously for five days a week on an open electronic limit order book system (GLOBEX) that is accessible by off-floor traders as well as by a number of market makers. GLOBEX is an international, automated order entry and matching system, which has a network extending to 10 financial centers, including New York, Chicago, London, and Tokyo. Trading on GLOBEX starts on Sundays at 5:00 p.m. Central Standard Time (CST) and ends on Fridays at 3:15 p.m. CST. On Mondays through Thursdays, trading stops at 3:15 p.m. CST and restarts at 3:30 p.m. CST. There is also a daily maintenance shut-down from 4:30 p.m. CST to 5:00 p.m. CST on Mondays through Thursdays.
} 
to span the occurrence of scheduled news announcements.

Two more points are in order regarding the choice of the dataset. First, in line with Birru and Figlewski (2012), we employ best bid and ask quotes rather than transaction prices because only rarely do we observe simultaneous transaction prices for a large number of different contracts. This problem becomes particularly pronounced for the further out-of-the-money options and for options with longer maturities and it precludes us from performing our analysis on transaction prices. On the other hand, the best option quotes are available at all points in time and they are continuously updated whenever the state of the order book changes. Moreover, we confirm that our quotes are companied with a large size relative to the trading volume and hence they are informative. Chan et al. (2002) find that option quotes can be more informative than trades. We discuss this issue further in Section 3. Second, the chosen time period contains both the mid 2007-2010 recent crisis period as well as the previous non-crisis one. Therefore, we will be able to check whether the number as well as the nature of jumps in option market differs over turbulent and non-turbulent periods.

CME offers two kinds of American style E-mini options which differ by their expiration months. Quarterly options expire in March, June, September, and December whereas serial options expire in January, February, and April. The underlying E-mini futures trades on quarterly expiries. Quarterly options are written on the E-mini that expires on the same day as the option. Serial options are written on the futures contract which has a maturity nearest to the option contract's. We match intra day options quotes with the simultaneously recorded underlying futures quotes and we discard observations for which this matching is not possible to avoid problems arising from non-synchronous underlying and option quotes. We also discard in-the-money option quotes because these options are highly illiquid (see Neumann and Skiadopoulos, 2013, for a similar approach). 
We apply a number of filters to the quotes of any given contract so as to minimise the impact of microstructure noise which is likely to contaminate the quotes data. In particular, we apply the Barndorff-Nielsen et al. (2009) filtering criteria commonly used in the market microstructure literature. First, we replace bid and ask quotes with identical time stamps by their median bid and ask quotes for this time stamp. Second, we discard quotes for which the bid-ask spread is negative. Third, we discard quotes for which the bid-ask spread is "excessively" wide. We remove contract's quotes whose spreads are greater than 50 times the daily median spread. ${ }^{4}$ We also discard quotes that are likely to represent outliers with respect to the midpoint quote. To this end, at any point in time where there is a quote, we compute the difference between the time $t$ observation and the median midpoint of bid and ask quotes of the 25 observations preceding and 25 observations following the time $t$ observation. We then calculate the daily mean of these differences. For any given day, we discard the observations which deviate by more than 10 times from this daily mean.

Next, we group option contracts into buckets based on their strikes and maturities. This classification serves two purposes. First, it provides a sufficient number of observations for each strike and maturity; tracking prices for each single option contract at high frequencies is not feasible because not all strikes are traded. Second, it allows us to investigate whether the characteristics of discontinuous option price movements differ across strikes and maturities.

\footnotetext{
${ }^{4}$ The choice of any filtering criterion involves a trade-off between the amount of noise and valuable information to be removed. Admittedly, in the market microstructure literature, the choice of threshold values may be ad-hoc when it comes to applying data filters. More stringent criteria may also be applied. For instance, an alternative choice could be to first remove all contracts which correspond to zero option trading volume during the day, and then apply the standard Barndorff-Nielsen et al. (2009) filters to the quotes of the remaining contracts; we would like to thank the referee for pointing this out. However, even the bid-ask quotes corresponding to options with zero trading volume may contain useful information (Hiraki and Skiadopoulos, 2019). In addition, we group options in various categories depending on how close or far-away from the money they are. The average option trading volume conditional on the occurrence of a jump is smaller for close to-the-money rather than far-away from the money options (see Table 4). This provides further evidence that the detected jumps are not driven by liquidity concerns once option liquidity is proxied by the option trading volume. If trading volume was the driver of detected jumps, then the number of close-to-the-money option related jumps should be higher compared to the one of away from-the-money options. However, we find that the reverse holds in general (see Table 3).
} 
An "idiosyncratic" behaviour of the discontinuous movements of option prices may be expected given that trading different options serves different purposes, and hence they may enjoy a different clientèle across the spectrum of strikes and maturities. Bakshi et al. (2000) and Sheikh and Ronn (1994) document such an idiosyncratic pattern in the call and put option raw returns, yet without isolating the jump component. In fact, the S\&P 500 E-mini futures options is currently populated by off-floor traders as well as by a number of market makers. ${ }^{5}$

We follow Bollen and Whaley (2004) and group option quotes according to their Black (1976) deltas into deep out-of-the-money (DOTM), out-of-the-money (OTM) and at-the-money (ATM) puts and calls; Panel A of Table 1 reports this classification (see Christoffersen et al., 2018, for a similar approach). The computation of option deltas requires estimates for the risk-free rate, the underlying volatility, and the simultaneously recorded underlying price. We assume risk-free rates to be constant through the trading day and we proxy them by the daily U.S. LIBOR rates with maturities one week, one month, two months up to 12 months obtained from the website of the St. Louis Fed. Whenever rates with maturities different from the ones covered by the data are required, we linearly interpolate between the rates of the two available adjacent maturities. We use the Black (1976) model to back out the implied volatility for each quote, and use it as the volatility input to calculate option's delta. ${ }^{6}$ Option prices as well as underlying prices are taken to be the mid-point of the bid and ask quotes. In addition to the delta dimension, we also classify option quotes according to their time to expiration into short-term, medium-term, and long-term options; Panel B of Table 1 reports this classification.

\footnotetext{
${ }^{5}$ Currently, six market makers operate in this market: Citadel Derivatives Trading LLC, Chicago Trading Company, Deutsche Bank Securities, Inc., Goldman Sachs, Timber Hill and Wolverine Trading LLC.

${ }^{6}$ Black (1976)'s model prices European style options. Its use to calculate the deltas and implied volatilities of the American style E-mini options is unlikely to introduce any error, though. This is because the early exercise premium is negligible given that we use ATM and OTM options with time-to-maturity less than 100 days (Barone-Adesi and Whaley, 1987). Hence, there is no loss in accuracy from using the computationally less expensive Black (1976) model. Using Black (1976)'s model does not assume that this model prices the options accurately. The Black (1976) model merely serves to map option prices as a function of strikes space to option prices as a function of deltas.
} 
The delta and maturity classifications yield 18 distinct groups of option quotes which provide a parsimonious and accurate description of the structure of traded options.

For each one of these groups, we compute a time series of high frequency returns where each return is measured over a period of length $\Delta t$. To this end, we divide each trading day into $n_{d}=\frac{T_{d}}{\Delta t}$ subsamples where $T_{d}$ is the number of observations per day. Then, for each one of these subsamples we select the option quote with delta closest to the midpoint delta of the delta category under scrutiny. Based on this quote and the latest quote for the same contract (i.e. same strike price and expiration date) before the end of the subsequent interval, we compute the high frequency log option return for the delta category under scrutiny. This approach ensures that we compute option returns from the same contract. Then, we repeat the same process over the following subintervals. The application of this procedure to each subinterval and delta/maturity bucket, yields a series of high-frequency option returns for all 18 delta/maturity categories.

The empirical implementation of this scheme requires a choice for the subinterval $\Delta t$. The jump detection test to be employed assumes $\Delta t$ is arbitrarily small. Hence, it is desirable to choose the subinterval as short as possible. However, the more granular the sampling frequency is, the more the data are contaminated by microstructure noise which can distort the subsequent jump detection. Hence, in line with Andersen et al. (2000), we employ volatility signature plots of high-frequency option returns to select the "optimal" subinterval length. Volatility signature plots depict realized volatility as a function of the sampling frequency. In the absence of microstructure noise, realized volatility defined as the squared root of summed squared intraday returns, should be invariant to changes in the sampling frequency provided the data is sampled fine enough. Figure 1 shows the average daily realized volatility over our sample as a function of different subinterval lengths for the various delta levels for the short-term options. Volatility 
signature plots the other two maturity buckets can found in the Online Appendix. We can see that the realized volatilities diverge as the subinterval length approaches zero, and they start converging around the 10 minutes mark. Hence, we choose a subinterval length $\Delta t=10$ minutes. This choice yields between 52,627 to 64,755 return observations depending on the delta-maturity bucket.

\subsection{News announcement data}

We employ a list of scheduled U.S. macroeconomic news announcements which includes 11 news items. We obtain the exact timing of the releases and their corresponding survey forecasts from Bloomberg. On Fridays, Bloomberg surveys key market participants for their forecasts regarding the values of economic variables that will be released within the next week. The median of the survey is taken to be the forecast for the respective economic variable. Table 2 reports the announcement items and their timing. All scheduled announcements take place within our daily sampling interval from 7:00 a.m. CST to 14:45 p.m CST with most of them being released at 7:30 a.m. on a monthly basis. The only exception is the FOMC rate announcement on 8/10/2008 which occurred on 6:00 a.m.; we exclude this announcement because it took place outside of our defined trading day. In total, our sample contains 888 announcements and 751 days on which at least one scheduled announcement has been released.

Following Balduzzi et al. (2001), we consider news surprises to assess the impact of news announcements on option markets; in an efficient market, prices should not respond to information that has already been anticipated by market practitioners. Let $A_{i, t}$ denote the $i^{\text {th }}$ news item's actual figure released at time $t$ and let $F_{i, t}$ denote the forecast for this figure. Then, the surprise measure $S U R_{i, t}$ is defined as 


$$
S U R_{i, t}:=\frac{A_{i, t}-F_{i, t}}{\sigma_{i}}
$$

where $\sigma_{i}$ denotes the sample standard deviation of the surprise components $A_{i, t}-F_{i, t}$ for the $i^{\text {th }}$ news item. We standardize the news surprises to facilitate comparison across different news items. As news surprises measure the component of a news release which is unanticipated by the market, we will also refer to them as information shocks in what follows.

\subsection{Liquidity Measures}

Market liquidity is defined as the ability to buy or sell significant quantities of securities quickly at a low cost with little price impact. We compute two liquidity measures to proxy two important dimensions of the definition of market liquidity: the bid-ask spread and the option sizes ordered at the bid and ask prices. The bid-ask spread measures the cost of executing a trade for a given size, whereas the size variable measures the depth of the market (i.e. how many contracts are offered) at the best bid and ask price.

First, for each option delta and maturity category we compute the time $t$ standardised bid-ask spread $s B A_{t}$

$$
s B A_{t}=\frac{A s k_{t}-B i d_{t}}{\sigma(B A)}
$$

where $A s k_{t}\left(B_{i d_{t}}\right)$ denotes the bid (ask) quote of the contract used to compute the 10-minute option returns in Section 2.1 and $\sigma(B A)$ the sample standard deviation of the dollar bid-ask spread $B A$ of the respective delta and maturity category. We compute a standardised bid-ask spread because the bid-ask magnitude depends on the option's strike and maturity.

Second, we obtain the time $t$ quoted sizes $\left(\right.$ AskSize $\left._{t}\right)$ and $\left(\right.$ BidSize $\left._{t}\right)$ at the best ask and bid quotes, respectively, for each delta/maturity category. To this end, we retain separately 
the ask and bid sizes of each one of the quotes used to compute the option returns in Section 2.1.

\section{Jumps in Option prices}

\subsection{Jump Test}

We employ the Lee and Mykland's (LM, 2008) jump detection test to test whether there are any jumps in option prices. Compared to competing approaches, the LM test has the advantage that it detects both the occurrence and the timing of jumps (see Dumitru and Urga (2012) for a review of jump detection tests). It does so by checking each recorded change in the asset price to conclude whether this is a jump or not. It relies on the idea that large movements in an asset price can either be caused by jumps or they could be realisations of a continuous yet highly volatile process. Hence, it adjusts the observed movements by the volatility of the continuous part of the stochastic price process. If a given adjusted movement is "too large", then this change is labelled a jump.

Let $S(t)$ denote the time $t$ asset price. In the absence of jumps, the stochastic evolution of $S(t)$ is represented by

$$
d \log S(t)=\mu(t) d t+\sigma(t) d W(t)
$$

where $W(t)$ is a Brownian Motion. $\mu(t)$ and $\sigma(t)$ are the drift and volatility stochastic processes, respectively, such that $d \log S(t)$ is an Itô process with continuous sample paths. In contrast, if jumps are present $S(t)$ is assumed to follow

$$
d \log S(t)=\mu(t) d t+\sigma(t) d W(t)+Y(t) d J(t)
$$


where $J(t)$ denotes a counting process that controls the arrival of jumps and $Y(t)$ denotes the jump size.

Assume there are $n$ (equidistant) observations of $S(t)$ available and $t \in[0, T]$, where $T$ denotes the total number of observations of any given time series of option returns. Then, the distance between observations $\Delta t$ is given by $\Delta t=\frac{T}{n}$. We test whether there is a jump at a particular time $t_{i} \in[0, T]$. The LM test standardises the log-return from $t_{i-1}$ to $t_{i}$ by the instantaneous volatility of the stochastic price process to account for its diffusive component. Thus, the LM test statistic is:

$$
\mathcal{L}(i) \equiv \frac{\log S\left(t_{i}\right)-\log S\left(t_{i-1}\right)}{\widehat{\sigma\left(t_{i}\right)}}
$$

where $\widehat{\sigma\left(t_{i}\right)}$ is estimated by the realized bipower variation (RBPV) using the past $K$ observations of $S(t)$. The RBPV estimator is given by

$$
\left.{\widehat{\sigma\left(t_{i}\right.}}^{2} \equiv \frac{1}{K-2} \sum_{j=i-K+2}^{i-1} \mid \log S\left(t_{j}\right)-\log S\left(t_{j-1}\right)\right]|| \log \left[S\left(t_{j-1}\right)\right]-\log \left[S\left(t_{j-2}\right)\right] \mid
$$

RBPV estimates the instantaneous volatility consistently even in the presence of jumps in the past $K$ observations. LM show that under the null of no jumps and as $\Delta t \rightarrow 0$, the distribution of $\mathcal{L}(i)$ approximately follows the distribution of a normally distributed random variable with mean 0 and variance $\frac{1}{c^{2}}$ with $c=\sqrt{2} / \sqrt{\pi}$. In contrast to this, LM show that in the presence of jumps as $\Delta t \rightarrow 0, \mathcal{L}(i)$ becomes very large. Hence, observing large values of the test statistic signifies the presence of jumps.

To assess how big the test statistic must be to indicate the presence of a jump at a certain significance level, LM employ the distribution of the maximum of the test statistic over $\mathcal{L}(i)$ under the null of no jumps. If the test statistic is greater than its maximum under the null of 
no jumps, it is highly unlikely that the observation in question was generated by a continuous process. As a consequence, one can base rejection of the null of no jumps on the rescaled and centred test statistic:

$$
\frac{|\mathcal{L}(i)|-C_{n}}{S_{n}}
$$

where $C_{n}=\frac{(2 \log (n))^{1 / 2}}{c}-\frac{\log (\pi)+\log (\log (n))}{2 c(2 \log (n))^{1 / 2}}, S_{n}=\frac{1}{c(2 \log (n))^{1 / 2}}$, and $n$ is the sample size. The null hypothesis of no jumps is rejected whenever $\frac{|\mathcal{L}(i)|-C_{n}}{S_{n}}$ exceeds the critical value $\beta^{*}$ obtained from a standard Gumbel cumulative distribution for a given confidence level $\alpha$ with $\beta^{*}$ such that $\exp \left(-\exp ^{-\beta^{*}}\right)=1-\alpha$, i.e. $\beta^{*}=-\log (-\log (1-\alpha))$.

To implement the LM test, one has to select a window size $K$ for the purpose of estimating instantaneous volatility. In line with LM, we choose $K$ to be the smallest integer in the interval between $\sqrt{252 \times \text { nobs }}$ and $252 \times$ nobs, where nobs denotes the number of observations per day. We determine the critical values by setting the Gumbel cumulative distribution function to a confidence level $\alpha=0.1 \%$. We choose such a conservative significance level to minimise the number of spuriously detected jumps; under the null hypothesis that there is no jump in any given subinterval, we expect to find a spuriously detected number of jumps equal to the number of observations times the chosen significance level. ${ }^{7}$

\subsection{Results}

We separately apply the LM test to the time series of the futures returns, and the option returns across the various delta and maturity categories. Table 3 reports the summary statistics (number of jumps, probability of a jump to occur, number of jump days, probability of a jump

\footnotetext{
${ }^{7}$ We have checked the robustness of the results with respect to changes in the significance level. We find that the results are qualitatively the same for different choices of the significance level ranging from $0.1 \%$ to $10 \%$. Results are not reported due to space limitations.
} 
day to occur, average jump size, and percentage of negative jumps as a fraction of total jumps) for each one of the delta and maturity categories. ${ }^{8}$ It also reports the same summary statistics for the underlying futures.

We can see that option prices jump. The number of jumps varies substantially across the delta and maturity buckets. With respect to the delta dimension, the DOTM calls and puts exhibit the greatest number of price jumps. With respect to the options maturity dimension, we can see that short maturity options exhibit more jumps than the longer maturity ones for all moneyness levels but OTM and DOTM calls. The documented heterogeneity of option jumps across moneyness and maturities is consistent with the fact that a number of traders with different motives trade in this market. Regarding the option's price jump size, we can see that this is negative on average and large (e.g., up to a $63 \%$ jump) with short-term options exhibiting substantially larger jump sizes than longer-term ones. The findings also suggest that downward option price jumps occur more often than upward jumps for almost all moneyness levels and maturities. Exact binomial tests reveal that the probability of observing a negative jump is significantly greater than $50 \%$ at the $5 \%$ significance level for most delta and maturity categories.

Finally, we compare the number of identified jumps in the price of the underlying asset to the number of identified option price jumps. We can see that the number of identified jumps in the underlying's price remains fairly constant across maturities; this is in contrast to the option price jump case. Furthermore, we can see that in most cases, the number of underlying jumps is less than the number of option price jumps. This finding has implications with respect to the question how jumps are transmitted to option prices. It indicates that option price jumps

\footnotetext{
${ }^{8}$ Jump sizes are defined to be the realized returns that have been identified as a jump. Note that strictly defined, these returns are the sum of the drift, diffusive, and jump component. Measuring the exact jump size would require disentangling the drift and diffusive component from the realized return. This is beyond the scope of this paper.
} 
cannot be solely attributed to simultaneous jumps in the price of the underlying asset. We investigate this relation further in the next section.

Two remarks are in order at this point regarding the credibility of our results. First, the employed best bid and ask quotes are reliable because they are associated with much larger sizes than the typical trading volume. Table 4 provides evidence for this. It shows the unconditional average size available at the best bid and ask price, as well as conditional on detecting a jump for the short, medium and long term options (Panels A, B, C, respectively). Additionally, the average trading volume per 10-minute interval is reported. We can see that on average, the best bid and ask sizes are much greater than the typical 10-minute trading volume. Therefore, the bid and ask quotes are on average able to accommodate the typical 10-minute trading activity. Second, the LM statistic is a conservative test in the sense that it captures large jumps. This is ensured by the construction of the test statistic as well as by the low significance level we have employed. Therefore, detected jumps are unlikely to be a manifestation of noise.

\section{Drivers of option price jumps}

\subsection{Jumps in the underlying factors}

Option pricing theory states that option prices are determined by the price of the underlying asset and its volatility by a no-arbitrage argument. In this section, we explore whether the detected jumps in option prices are due to jumps in the price of the underlying asset and/or its volatility. We define co-jumps (or simultaneous jumps) as the jumps which occur within the same 10 minute interval. If jumps in option prices arise from jumps in the underlying asset price and volatility, then one would expect these underlying factors to jump simultaneously with the prices of the ATM options (co-jumps); ATM options have the greatest (absolute) deltas and 
vegas. In addition, co-jumps across strikes may be observed. In the case where the underlying and or volatility co-jumps with DOTM options, options closer to at-the-money should jump as well because their deltas and vegas are greater than the DOTM options' ones.

To identify whether option prices co-jump, Figure 2 reports the frequency of different cojump events for the short, medium and long-term maturity buckets, respectively. A co-jump event is characterized by the number of concurrent jumps (i.e. jumps within the same 10 minute interval) across options of different delta levels and the underlying. The figures depict for each maturity bucket how often options of one, two, three,..., six delta categories and/or the underlying have jumped simultaneously. In particular, the case where the number of concurrent jumps is one refers to an idiosyncratic option price jump in one of the delta categories or in the underlying price. We can see that co-jumps are rare. The vast majority of option price jumps are not accompanied by simultaneous jumps in option prices of other delta buckets or by a simultaneous jumps in the underlying asset. ${ }^{9}$

Yet, the mere evidence that most option price jumps are idiosyncratic across delta categieries does not rule out the possibility that some of the detected option price co-jumps are still due to price and/or volatility jumps. For instance, a jump in the underlying price may yield a jump in the ATM option price but not necessarily a jump in OTM and DOTM option prices. Similarly, cases where only ATM calls and puts jump simultaneously might be attributed to volatility jumps as these moneyness categories are more sensitive to changes in volatility than OTM/DOTM options. To investigate this further, we examine which delta categories and/or the underlying asset jump simultaneously (termed composition of co-jump events).

\footnotetext{
${ }^{9}$ We have also broadened the definition of co-jumps in two ways. First, we consider 10-minute intervals adjacent to the detected jump. Focusing at each maturity and moneyness bucket separately, we calculate the number of jumps that the remaining categories exhibit 10-minutes before, simultaneously and 10-minutes after we detect a jump in the bucket under consideration. We confirm that the detected jumps are predominantly idiosyncratic. In the vast majority of the cases when we detect a jump in a specific bucket at time $\mathrm{t}=0$, we do not detect any jumps 10 minutes before $(t=-10)$, simultaneously $(t=0)$ or 10 minutes after $(t=+10)$ in the remaining categories. This corroborates further that the detected jumps are predominantly idiosyncratic. These results are reported and discussed also in the Online Appendix.
} 
Figure 3 shows the composition of co-jumps for the short, medium, and long-term maturities, respectively. We can see that the already small number of detected co-jumps is spread across various delta and delta/underlying combinations; they do not show up in the ITM options and underlying category or in the ATM put and call options which would be evidence for options cojumping with the underlying factors. In addition, there is no specific pattern of the composition of co-jumps across maturities. Therefore, co-jumps do not cluster in a particular combination of delta categories.

To sum up, our findings suggest that option price jumps are not due to jumps in the underlying price and its volatility. Moreover, the presence of idiosyncratic jumps in option prices implies that there is not a common factor that explains the variability of the crosssection of jump induced option returns. This is not at odds with the literature which finds that there are common factors in the cross-section of total (defined to be the sum of diffusive and discontinuous) option returns though (e.g., Christoffersen et al. (2018)).

\subsection{Information events as drivers of option price jumps}

We explore further the drivers of jumps in option prices. From a theoretical perspective, news announcements can make option prices jump. This is because heterogeneous beliefs (Shefrin, 2001; Buraschi and Jiltsov, 2006; Friesen et al., 2012) and market sentiment (Han, 2008; Lemmon and $\mathrm{Ni}, 2011$ ) are related to the slope of the implied volatility curve. ${ }^{10}$ Given that certain news may affect these factors drastically, one might expect these news effects to be transmitted to the slope of the implied volatility skew in a jump-like fashion. This will be manifested as jumps in option prices. Motivated by these considerations, we investigate whether jumps in option prices can also be related to macroeconomic news announcements.

\footnotetext{
${ }^{10}$ On any given point in time and for any given option expiry, the implied volatility curve is defined to be the relation between the options implied volatilities and their respective strikes.
} 
To investigate to what extent detected option price jumps are related to scheduled macroeconomic news announcements, we match the detected jumps with the the release of scheduled news announcements events presented in Section 2.2. We define that an identified jump is related to a specific news announcement if the jump has occurred within \pm 10 minutes of the respective announcement.

Panel A of Table 5 reports the conditional probabilities $P(N e w s \mid J u m p)$ and $P($ Jump $\mid$ News $)$ to detect the relation between the detected jumps and all considered macroeconomic news. $P($ News $\mid$ Jump $)$ shows the fraction of detected jumps associated with news announcements. $P($ Jump $\mid$ News $)$ shows the fraction of news associated with jumps, i.e. it denotes the probability that a news announcement triggers a jump.

Regarding $P($ News|Jump), we can see that $14.35 \%$ to $28.50 \%$ of detected jumps are linked to the scheduled release of macroeconomic news depending on the delta and maturity category. The number of news-related jumps in the underlying asset differs substantially from the options ones. This indicates that the previously documented segmentation of option and underlying price jumps prevails around scheduled news announcements, as well. Regarding $P(J u m p \mid N e w s)$, we can see that the probability of news yielding jumps is greater for DOTM calls and puts and it is greatest for short-term options; $P($ Jump $\mid$ News $)$ ranges from $1.35 \%$ to $6.86 \%$. Hence, it is more probable that a news release will yield an option price jump for shorter than for longer term options.

To shed more light on the relative importance of the individual news items reported in Section 2.2, we report the probabilities $P($ News $\mid$ Jump $)$ and $P(J u m p \mid N e w s)$ for each news item separately in Table 5, Panels B and C, respectively. Regarding $P(N e w s \mid J u m p)$, the nonfarm payrolls (NFP) report and the initial jobless claims (IJC) are associated with detected jumps more than the other releases are. In particular, the NFP 
report is associated with up to $14.13 \%$ of the detected jumps whereas IJC is associated up to $11.51 \%$ of the detected option price jumps.

Regarding the probability $P(J u m p \mid N e w s)$ that a specific news release will trigger a jump, we can see that the NFP report is the news item that is most likely to trigger an option price jump, among all individual news items. For certain delta categories of short-term options, a NFP release results in a jump in more than $20 \%$ of all cases. This is in line with the existing literature on jumps and news announcements effects in financial markets which documents that the NFP report is the most influential scheduled news announcement (e.g., Andersen and Bollerslev (1998)). Interestingly, $P($ Jump $\mid$ News $)$ is masked when news releases are aggregated; it increases from $1.4 \%$ - $7 \%$ when all news items are considered jointly to $20 \%$ when NFP is considered in isolation.

\subsection{Information shocks as sources of option price jumps}

Up to now our findings suggest that a fraction of option price jumps is triggered by information events. However, one may hypothesize that not only the fact that new information is released but also the content of the released information itself explains the occurrence of jumps.

We examine this hypothesis by statistically linking the occurrence of detected option price jumps to the content of the released scheduled macroeconomic news. To this end, we employ a logistic regression methodology (see Jiang et al. (2011) for a similar approach). Lee (2012) shows that this approach allows drawing inference on the determinants of the unobservable stochastic jump intensity of the continuous time jump process even when one employs discrete time data (option returns and jump determinants). We estimate the following specification

$$
P\left(\text { Jump }_{t} \mid \text { News }\right)=\frac{1}{1+\exp \left(-c-\sum_{j=1}^{11} \theta_{j}\left|S U R_{j, t}\right|\right)}
$$


where $j \in \quad\{$ NFP, CCI, CPI, DGO, FOMC, GDP, IJC, LI, NHS, PPI, RSA $\} \quad$ and $P\left(\right.$ Jump $_{t} \mid$ News $)$ denotes the probability of an option price jump to occur conditional on a scheduled macroeconomic announcement taking place; ex post it takes a value of 1 when there is a jump at the announcement time $t$, and 0 otherwise. This conditioning is necessary because the values of the macroeconomic surprises variables are only available at announcement times which implies that equation (8) can only be estimated for observations coincident with announcement times. Therefore, a logistic regression unconditional on any news event cannot be conducted.

A few remarks are in order at this point regarding the estimation of equation (8). For any given delta and maturity category, the number of option price jumps that can be linked to the concurrent release of scheduled news, is too small to estimate equation (8) accurately for each delta/maturity category separately. To increase the statistical accuracy of our estimates, we pool observations across different delta levels and estimate equation (8) once for each maturity category. We also only incorporate announcement items which exhibit at least one non-zero surprise matched with a concurrent option price jump. Pooling across different delta categories is not expected to affect our results for two reasons. First, the results of the analysis in Section 4.2 do not reveal any major differences across deltas with respect to the question which news items are most important in explaining option price jumps. Second, we use absolute surprises and a binary jump indicator variable so that the expected sign of the $\theta_{j}$ is the same (positive) for all delta categories.

Panel A of Table 6 reports the estimation results for equation (8). In line with the evidence from Section 4.2, NFP surprises have a significant positive impact on the probability of a jump to occur in short and medium-term options. There is no significant effect of NFP on the probability of a jump to occur in long-term options. For these options instead, GDP as well 
as retail sales less auto surprises have a positive impact on the jump probability. However, in general the evidence for a strong relation between news surprises and option price jumps appears to be rather weak; only a small number of coefficients in equation (8) turns out to be significant. The results from the logistic regression approach suggest that the triggering of option price jumps by news announcements reported in Section 4.2 is not due to the news content, i.e. due to the fact that new information is being impounded into prices. This implies that option price jumps are primarily driven by other determinants. We explore this further in the next section.

\subsection{Illiquidity as a driver of option price jumps}

As a final source of option price jumps, we investigate any effect of illiquidity on the associations between jumps and news releases. Christoffersen et al. (2018) find that option illiquidity reduces current option prices and predicts future option price increases. Hence, rapid movements in option liquidity might result in jumps in option prices.

First, we examine the effect of changes of liquidity on the probability of news related option jumps. We re-estimate equation (8) by augmenting the set of covariates by the relative bid-ask spread, quoted size at the best bid price, and quoted size at the best ask price (see Section 2.3). We estimate

$$
P\left(\text { Jump }_{t} \mid \text { News }\right)=\frac{1}{1+\exp \left(-c-\sum_{j=1}^{11} \theta_{j}\left|S U R_{j, t}\right|-\sum_{k=1}^{3} \gamma_{k} I L_{k, t-1}\right)}
$$

where $I L_{k, t-1}$ denotes the time $t-1$ value of the $k^{\text {th }}$ liquidity variable. We do not include variables related to the liquidity of the underlying futures market in equation (9). This is because the previous analysis showed that jumps in the option market are not related to jumps in the underlying market. 
Panel B of Table 6 reports the estimation results for the model shown in equation (9). We can see that the coefficients of the standardised bid-ask spread are positive and highly significant for all maturity categories. The coefficients of the bid and ask sizes are negative, yet they are significant only sporadically. These results show that option price jumps are triggered by option market liquidity dry ups. Most importantly, almost all news surprise variables become insignificant after adding the liquidity variables to the model. Hence, after controlling for liquidity in the option market, the content of the considered news announcements has almost no power in explaining option price jumps. Similarly, Jiang et al. (2011) and Boudt and Petitjean (2014) find that illiquidity predicts jumps in bond and equity prices beyond information shocks induced by macroeconomic news announcements.

Our findings suggest that it is liquidity and not the content of the news surprises that drives the occurrence of option price jumps around announcements. To confirm this visually, for each option delta and maturity category we compute the time $t$ relative bid-ask spread $B A_{t}$

$$
B A_{t}=\frac{A s k_{t}-B i d_{t}}{\left(A s k_{t}+B i d_{t}\right) / 2}
$$

where $A s k_{t}\left(\operatorname{Bid}_{t}\right)$ denotes the bid (ask) quote of the contract used to compute the 10-minute option returns in Section 2.1. We compute a relative bid-ask spread because the bid-ask magnitude depends on the option's strike and maturity (see Christoffersen et al., 2018 for a similar choice). Figure 4 shows the median relative bid-ask spreads for a number of time subintervals around the news related jumps (10 minutes before the jump up to 60 minutes after the jump) across the various moneyness levels for the case of the short maturity options. We can see that the spread increases significantly on the jump time (point zero in the graph) in the case of the short maturity options; the pattern is similar for the other two maturity buckets and it is not reported due to space limitations. 
The fact that the bid-ask spread increases on the announcement day can be explained by considering asymmetric information among traders as a key determinant of quoted bid-ask spreads in limit order book markets (Handa et al., 2003). Option traders widen the quoted bid-ask spreads and thus, they decrease market liquidity just before the news announcement to avoid the risk of trading with traders with superior information about the upcoming information event. This practice extends the evidence from index-linked limit order book markets (Erenburg and Lasser, 2009) and it is highly relevant to options markets because these are commonly considered to be a natural setting for informed traders (Chan et al., 1995; Easley et al., 1998; Chakravarty et al., 2004; Pan and Poteshman, 2006, and references therein).

To shed more light on the above information asymmetry explanation for the link between news related jumps and liquidity, we investigate the nature of the information asymmetry. In the terminology of Kim and Verrecchia (1997), information asymmetry can be either "preannouncement" and / or "event-period" private information. The former stems from some information leakage which is not available to all traders. The latter type of information asymmetry stems from the fact that some traders have better skills in processing information when this is announced publicly thus effectively making it private information (Kim and Verrecchia, 1994). In the case where there is no pre-announcement asymmetric information, there should be no relation between trading volume and price changes.

We plot the distribution of traded volume on the jump time, as well as 10 minutes before the jump, to check whether option jumps are driven by pre-announcement private information. Figure 5 plots the frequency (in \%) distribution of the option volume for any given delta bucket for the short-term options. We consider the percentage frequency for three volume buckets of zero, 10, and more than 10 contracts. Similar plots for medium- and long-term options can be found in the Online Appendix. We can see that the vast majority of news related jumps 
is accompanied by zero volume. This indicates that there is no private information due to information leakage prior to scheduled news announcement (see Ederington and Lee, 1995 for similar evidence); if it were, then trading activity should take place prior to the announcement. Instead, traders increase their bid-ask spreads in the fear that they will interact with investors who are better skilled in processing information once the announcement is released; the increase in bid-ask spreads arises due to order cancellation or bid-ask spread revision. ${ }^{11}$

We confirm the absence of pre-announcement private information in the case of news-related jumps by re-estimating (9) by including the trading volume as an additional regressor, i.e.

$$
P\left(\text { Jump }_{t} \mid \text { News }\right)=\frac{1}{1+\exp \left(-c-\sum_{j=1}^{11} \theta_{j}\left|S U R_{j, t}\right|-\sum_{k=1}^{3} \gamma_{k} I L_{k, t-1}-\beta V o l_{t-1, t}\right)}
$$

where $V_{o l} l_{t-1, t}$ denotes the total trading volume in the respective moneyness/maturity bucket from time $t-1$ to $t$. Panel A of Table 7 reports the estimation results for the model shown in equation (11). We can see that the option trading volume variable is insignificant as expected. This confirms that the observed increase in options bid-ask spreads is not due to pre-announcement private information.

To robustify the evidence on the link between illiquidity and option price jumps, we explore the determinants of option price jumps which are not related to scheduled news announcement times. We estimate the following logistic regression based on a pooled (across delta categories) sample of non-news related observations

$$
P\left(J_{u m p} \mid \text { No News }\right)=\frac{1}{1+\exp \left(-c-\sum_{k=1}^{3} \gamma_{k} I L_{k, t-1}-\beta V o l_{t-1, t}\right)}
$$

\footnotetext{
${ }^{11}$ The information asymmetry explanation for the detected jumps cannot not explain on its own the documented non-synchronous occurrence of jumps across strikes. This is because an informed trader may choose which strike to trade by taking other factors into account such as leverage, transaction costs, commissions etc. (Chakravarty et al., 2004, and references therein). The relative importance of these factors may also change through time, thus also partially explaining the idiosyncraticity of the detected jumps.
} 
Panel B of Table 7 reports the estimation results. We can see that non-news related jumps are strongly related to increases in illiquidity in option markets. This result holds for all liquidity measures considered. Our findings suggest that option market illiquidity is by far the most important driver of option price jumps. Interestingly, the option trading volume is significant now. The significance of the liquidity and volume variables reveals an explanation for the occurrence of the no-news related jumps. In this case, the increase in bid-ask spreads cannot be attributed to an information asymmetry story because of the nature of detected jumps. Instead, it is attributed to the fact that the increase in trading activity fills in orders and as a result the bid-ask quotes that are further down in the order book advance to the top of the order book.

\section{$5 \quad$ Further robustness analysis}

We provide further robustness tests. First, we conduct a subsample analysis. Second, we consider the relation of detected jumps with unscheduled news announcements. Third, we use a different index options dataset, namely data on S\&P cash-settled index options.

\subsection{Subsample analysis}

We investigate the existence of option price jumps over two subsamples. We divide the entire sample period from 1 May 2005 to 31 December 2010 into a non-crisis and a crisis period spanning 1 May 2005 to 31 July 2007 and 1 August 2007 to 31 December 2010, respectively; August 2007 is typically considered to mark the beginning of the global credit crisis. We then recompute the jump and jump-news statistics as in Section 4.2, separately, for both the non-crisis and crisis sub-samples.

Panels A and B of Table 8 report summary statistics for the detected option price jumps 
for the non-crisis and crisis period, respectively. We can see that the jump frequencies in the non-crisis and crisis subsamples are of similar magnitude, for any given maturity category. Hence, jumps in option prices exist regardless of the general market conditions contradicting conventional perception that jumps mainly occur over crisis periods.

Panels A and B of Table 9 report the summary statistics on the relation between jumps and all scheduled macroeconomic news announcements for the non-crisis and the crisis periods, respectively. The comparison of the non-crisis to the crisis figures reveals an interesting pattern. The association of jumps and news is stronger in the crisis than in the non-crisis sub-sample. Both the probability of news to cause a jump as well as the fraction of jumps related to news announcements are substantially greater in the crisis than in the non-crisis sub-sample for almost all delta and maturity categories. In the most pronounced case (short-term DOTM calls), the probability of a news announcement triggering a jump is almost three times greater in the crisis than in the non-crisis sub-sample. This suggests that option markets have been more sensitive to the release of macroeconomic news announcements during the crisis than the non-crisis periods.

Finally, Panels A and B (C and D) of Table 10 report $P($ News $\mid$ Jump) and $P($ Jump $\mid$ News $)$ disaggregated by news items for the non-crisis (crisis) period. We can see that the results are in line with the results aggregated over all announcements as well as with the results over the full sample (Section 4.2). The NFP report as well as the IJC turn out to be the news items most associated with jumps both in the non-crisis and crisis periods. Also, the jump-news relation appears to be stronger in the crisis than in the non-crisis period.

The more pronounced clustering of option price jumps around the release of scheduled news in the crisis period also raises the question whether the news content is more powerful in explaining the occurrence of option price jumps in the crisis period. In fact, the empirical 
evidence in the context of spot markets, suggests that macroeconomic news surprises affect equity prices differently depending on the state of the business cycle (Andersen et al., 2007). This diverse response of jumps to news surprises across different states of the economy might also carry over to option markets.

To shed more light on this question, we re-estimate the logistic regression equation (11) on the crisis sub-sample. Table 11 reports the estimation results. Surprisingly, even though the association between news events and option price jumps has been found to be stronger in the crisis period, the explanatory power of the content of news surprises for option price jumps turns out to be low. Only three news surprise coefficients turn out to be significant.

As a further robustness check, we examine the dynamics of option market illiquidity over the non-crisis and crisis periods. Given that the likelihood of option price jumps has been found to be similar across the crisis and non-crisis sub-sample, one would expect the dynamics of option market illiquidity not to differ either. This is because our results over the full sample period suggest that the arrival of option price jumps is mostly driven by option market's illiquidity.

Figure 6 depicts the evolution of the daily average relative bid-ask spread for short-term options of the various delta categories. Similar plots for the remaining maturities can be found in the Online Appendix. We can see that the dynamics of option market illiquidity are comparable in the non-crisis and crisis periods. In particular, the dynamics of illiquidity do not appear to be any more erratic in the crisis sub-sample than in the non-crisis sub-sample. This is in line with the previous finding that option price jumps are equally likely in the non-crisis and crisis periods provided option price jumps are driven by option market illiquidity. Hence, we conclude that the results from the sub-sample analysis further confirm that it is liquidity and not the content of news shocks that drive jumps in option prices. 


\subsection{Unscheduled news announcements}

As a second robustness check, we extend the set of information shocks considered in our analysis. The vast majority of papers studying news announcement effects on financial markets have focussed on the analysis of scheduled news announcements (Andersen et al., 2007; Lahaye et al., 2011). Our list of scheduled macroeconomic news described in Section 2.2 includes the news items most commonly used in the existing literature and it can be regarded as a comprehensive list of the universe of scheduled information shocks. However, information shocks might also arise from the release of unscheduled news.

In what follows, we match detected option price jumps with a set of unscheduled news announcements. The list of announcements considered is taken from Jiang et al. (2012) and it includes a total of 137 unscheduled announcement items. The selection of these items has been based on the chronology of significant events of the California Department of Finance, the crisis time line provided by the Federal Reserve Bank of St. Louis, and nthe European crisis time line provided by Bloomberg.

Tracing the exact intra-day timing of an unscheduled news announcement is not feasible because different data sources provide a different timing. Hence, we match detected option price jumps with unscheduled news announcements on a daily level. In particular, we define an unscheduled news day to be the day on which at least one unscheduled news announcement has been released and we compute the fraction of jump days that are equal to unscheduled news days. A remark is in order at this point. 72 of the unscheduled news days in our sample coincide with scheduled news days as well. Consequently, it is not possible to unambiguously attribute a jump day to either unscheduled or scheduled news on these days. Therefore, we only retain unscheduled news days on which there has been no release of scheduled news.

Table 12 reports the results of the resulting jump day-unscheduled news day matching. We 
can see that unscheduled news play a minor role in explaining option price jump days. Only up to $6 \%$ of the detected option price jump days might be attributed to the release of unscheduled news across all delta/maturity categories. Hence, we can conclude that by focussing on the set of scheduled news announcements employed in Section 2.2, we do not ignore any relevant effect arising from unscheduled news announcements. Furthermore, the unimportance of unscheduled news for explaining option price jumps is consistent with liquidity being the most important jump determinant. This is because unscheduled news announcements occur unexpectedly by definition and thus they cannot adversely affect market liquidity through the informed trading channel described in Section 4.4.

\section{The case of highly liquid S\&P 500 index options}

We extend our analysis by considering S\&P 500 index options. S\&P 500 index options are actively traded on CBOE from 8:30 a.m. to 3:15 p.m. Chicago time over our entire sample period, i.e. at times beyond 7:30 a.m. where most of scheduled macroeconomic news announcements occur. The E-mini S\&P 500 options market, which we have used up to now, has the advantage that it is a 24 hour market and hence, it allows us to match scheduled macroeconomic news announcements to the detected jumps. In the case of S\&P 500 index options, we capture, even imperfectly, the effect of pre-market opening news announcements by considering overnight returns.

We obtain OPRA trade and quote data on S\&P 500 index options from TickData over the period 1 January 2005 to 31 December 2010. This data set includes bid and ask quotes, quote size, trade price and trade size time-stamped at least down to the second. We sample quotes from 8:30 a.m. to 14:45 p.m. CST. We apply the same filters as the ones used in our main analysis to remove noisy data and minimise the effect of microstructure noise (Barndorff-Nielsen 
et al., 2009). Next, we group options into the maturity and delta buckets shown in Table 1. To allocate each intraday observation to a moneyness bucket, we estimate implied volatility and delta using the Black (1976) model. We consider the mid-quote of the shortest E-mini futures contract as the underlying asset price of S\&P 500 index options; this is different from the underlying asset price of E-mini options where we use the mid-quote of the E-mini futures contract with the same or nearest maturity to the option contract. To avoid non-synchronicity concerns, we match the shortest E-mini S\&P 500 futures quotes to simultaneous S\&P 500 index option quotes. We also proxy intraday interest rates with the daily linearly interpolated U.S. LIBOR rates (LIBOR maturities between one week and one year).

Once we group our options data, we calculate 10-minute returns for the underlying shortest e-mini S\&P 500 futures contract and each one of the 18 options buckets. Given that the majority of the news announcements occurs at 7:30 a.m. CST, i.e. before the S\&P 500 index option market opens, we also calculate overnight returns, in addition to the over-day returns. We calculate overnight returns, using quotes from the last 10-minute interval of the previous day and the first 10-minute interval of the current day. Subsequently, we apply the LM test to detect jumps.

Overall, we find that jumps are idiosyncratic in the sense that they do not jump simultaneously (i.e. with the same 10-minute interval), just as was the case with the E-mini options market. We also document that a fraction of the detected jumps is linked to scheduled macroeconomic news announcements; in $4.7 \%$ to $10.8 \%$ of the cases a news announcement triggers a jump. Finally, we find that illiquidity is not the key determinant of option jumps. In fact, the content of news announcements (and not illiquidity variables) affects significantly the probability of a news-related S\&P 500 option price jumps. Moreover, the relative bid-ask spread is stable around news related jumps. More information and results can be found in the Online 
Appendix.

\section{Conclusions}

We provide first-time evidence on the characteristics and drivers of option price jumps by employing high-frequency index options quotes from a limit order book market. We find that E-mini S\&P 500 option price jumps are rare, sizeable, do not occur simultaneously across strikes and maturities, and are uncorrelated with jumps in the underlying futures price. On the other hand, only $14 \%$ to $28 \%$ of the detected option price jumps are associated with scheduled releases. However, even though the occurrence of news announcements triggers a fraction of option price jumps, the specific news content does not. Instead, we find that the option market's liquidity measured by option bid-ask spreads drives option price jumps. Moreover, we document that the increase in option bid-ask spreads is not explained by trading activity.

Our findings have three implications. First, jumps in E-mini S\&P 500 option quotes are idiosyncratic. This extends previous evidence by Sheikh and Ronn (1994) and Bakshi et al. (2000) who find that total, i.e. the sum of continuous and discontinuous, option returns exhibit heterogeneous dynamics across traded option contracts. Second, the E-mini S\&P 500 option market is segmented from the underlying market in terms of the discontinuous changes in asset prices. This complements the findings of Bakshi et al. (2000) who find that option prices do not always move in line with the underlying asset price. Third, the fact that liquidity rather than the content of information shocks drives E-mini S\&P 500 option price jumps, may also be explained by the fact that illiquidity increases prior to news announcements so that traders protect themselves against informed traders. Our evidence also suggests that the informational advantage in this option market is not due to some private information as a result of information leakage prior to the announcement. Instead, the information asymmetry stems from the fact 
that some investors may be more skilled in processing the released information than others, thus making market makers to increase bid-ask spreads; this increase is manifested as an option price jump. Interestingly, in the case of the $\mathrm{S} \& \mathrm{P} 500$ index options market, where we also find sizable and idiosyncratic price jumps, we document that the key determinant of option jumps is the content of news announcements rather than illiquidity.

Our findings open four avenues for future research. Our analysis can be extended to other option markets to check whether our findings hold there, as well. It would also be worth exploring whether existing option pricing model can generate the documented jump patterns in option prices. In the case they do not, one should look into developing a limit order book market microstructure model that generates idiosyncratic jumps in the cross-section of option prices. Third, our results further support the call for incorporating option market liquidity risk into option pricing theory (Christoffersen et al., 2018). Finally, the presented evidence is of interest to exchanges for the purposes of setting option margins. 


\section{References}

Andersen, T. G. and Bollerslev, T. (1998). Deutsche mark-dollar volatility: Intraday activity patterns, macroeconomic announcements, and longer run dependencies, Journal of Finance 53: 219-265.

Andersen, T. G., Bollerslev, T., Diebold, F. X. and Laby, P. (2000). Great realisations, Risk March 2000: 105-108.

Andersen, T. G., Bollerslev, T., Diebold, F. X. and Vega, C. (2007). Real-time price discovery in global stock, bond and foreign exchange markets, Journal of International Economics 73: 251-277.

Augustin, P., Brenner, M. and Subrahmanyam, M. G. (2019). Informed options trading prior to takeover announcements: Insider trading?, forthcoming in Management Science.

Bailey, W., Zheng, L. and Zhou, Y. (2014). What makes the VIX tick?, Working Paper, Cornell University .

Bakshi, G., Cao, C. and Chen, Z. (2000). Do call prices and the underlying stock always move in the same direction?, Review of Financial Studies 13: 549-584.

Balduzzi, P., Elton, E. J. and Green, T. C. (2001). Economic news and bond prices: Evidence from the U.S. treasury market, Journal of Financial and Quantitative Analysis 36: 523-543.

Barndorff-Nielsen, O. E., Hansen, P. R., Lunde, A. and Shephard, N. (2009). Realized kernels in practice: Trades and quotes, Econometrics Journal 12: C1-C32.

Barone-Adesi, G. and Whaley, R. E. (1987). Efficient analytical approximation of American option values, Journal of Finance 42: 301-320. 
Berkman, H. (1996). Large option trades, market makers, and limit orders, Review of Financial Studies 9: 977-1002.

Birru, J. and Figlewski, S. (2012). Anatomy of a meltdown: The risk neutral density for the S\&P 500 in the fall of 2008, Journal of Financial Markets 15: 151-180.

Black, F. (1976). The pricing of commodity contracts, Journal of Financial Economics 3: 167179.

Bollen, N. P. B. and Whaley, R. E. (2004). Does net buying pressure affect the shape of implied volatility functions?, Journal of Finance 59: 711-753.

Boudt, K. and Petitjean, M. (2014). Intraday liquidity dynamics and news releases around price jumps: Evidence from the djia stocks, Journal of Financial Markets 17: 121-149.

Broadie, M., Chernov, M. and Johannes, M. (2009). Understanding index option returns, Review of Financial Studies 22: 4493-4529.

Buraschi, A. and Jiltsov, A. (2006). Model uncertainty and option markets with heterogeneous beliefs, Journal of Finance 61: 2841-2897.

Chakravarty, S., Gulen, H. and Mayhew, S. (2004). Informed trading in stock and option markets, Journal of Finance 59: 1235-1257.

Chan, K., Chung, Y. P. and Fong, W. (2002). The informational role of stock and option volume, Review of Financial Studies 15: 1049-1075.

Chan, K., Chung, Y. P. and Johnson, H. (1995). The intraday behavior of bid-ask spreads for NYSE stocks and CBOE options, Journal of Financial and Quantitative Analysis 30: 329346. 
Christoffersen, P., Goyenko, R., Jacobs, K. and Karoui, M. (2018). Illiquidity premia in the equity options market, Review of Financial Studies 31: 811-851.

Coval, J. D. and Shumway, T. (2001). Expected option returns, Journal of Finance 56: 9831009.

Driessen, J. and Maenhout, P. (2007). An empirical portfolio perspective on option pricing anomalies, Review of Finance 11: 561-603.

Dumitru, A.-M. and Urga, G. (2012). Identifying jumps in financial assets: A comparison between nonparametric jump tests, Journal of Business and Economic Statistics 30: 242255.

Easley, D., O'Hara, M. and Srinivas, P. (1998). Option volume and stock prices: Evidence on where informed traders trade, Journal of Finance 53: 431-465.

Ederington, L. H. and Lee, J. H. (1995). The short-run dynamics of the price adjustment to new information, Journal of Financial and Quantitative Analysis 30: 117-134.

Ederington, L. H. and Lee, J. H. (1996). The creation and resolution of market uncertainty: The impact of information releases on implied volatility, Journal of Financial and Quantitative Analysis 31: 513-539.

Erenburg, G. and Lasser, D. (2009). Electronic limit order book and order submission choice around macroeconomic news, Review of Financial Economics 18: 172-182.

Evans, K. P. (2011). Intraday jumps and US macroeconomic news announcements, Journal of Banking and Finance 35: 2511-2527.

Fornari, F. and Mele, A. (2001). Volatility smiles and the information content of news, Applied Financial Economics 11: 179-186. 
Friesen, G. C., Zhang, Y. and Zorn, T. S. (2012). Heterogeneous beliefs and risk neutral skewness, Journal of Financial and Quantitative Analysis 47: 851-872.

George, T. J. and Longstaff, F. A. (1993). Bid-ask spreads and trading activity in the S\&P 100 index options market, Journal of Financial and Quantitative Analysis 28: 381-397.

Gonçalves, S. and Guidolin, M. (2006). Predictable dynamics in the S\&P 500 index options implied volatility surface, Journal of Business 79: 1591-1635.

Han, B. (2008). Investor sentiment and option prices, Review of Financial Studies 21: 387-414.

Handa, P., Schwartz, R. and Tiwari, A. (2003). Quote setting and price formation in an order driven market, Journal of Financial Markets 6: 461-489.

Hiraki, K. and Skiadopoulos, G. (2019). The contribution of frictions to expected returns, Working Paper, Queen Mary, University of London .

Huh, S.-W., Lin, H. and Mello, A. S. (2014). Hedging by option market makers: Theory and evidence, Working Paper, University of Wisconsin at Madison .

Jackwerth, J. C. (1999). Option implied risk-neutral distributions and implied binomial trees: A literature review, Journal of Derivatives 7: 66-82.

Jiang, G. J., Konstantinidi, E. and Skiadopoulos, G. (2012). Volatility spillovers and the effect of news announcements, Journal of Banking and Finance 36: 2260-2273.

Jiang, G. J., Lo, I. and Verdelhan, A. (2011). Information shocks, liquidity shocks, jumps, and price discovery: Evidence from the US treasury market, Journal of Financial and Quantitative Analysis 46: 527-551.

Kandel, E. and Pearson, Neil, D. (1995). Differential interpretation of public signals and trade in speculative markets, Journal of Political Economy 103: 831-872. 
Kim, O. and Verrecchia, R. E. (1994). Market liquidity and volume around earnings announcements, Journal of Accounting and Economics 17: 41-67.

Kim, O. and Verrecchia, R. E. (1997). Pre-announcement and event-period private information, Journal of Accounting and Economics 24: 395-419.

Lahaye, J., Laurent, S. and Neely, C. J. (2011). Jumps, cojumps and macro announcements, Journal of Applied Econometrics 26: 893-921.

Lee, S. S. (2012). Jumps and information flow in financial markets, Review of Financial Studies 25: 439-479.

Lee, S. S. and Mykland, P. A. (2008). Jumps in financial markets: A new nonparametric test and jump dynamics, Review of Financial Studies 21: 2535-2563.

Lemmon, M. L. and Ni, S. X. (2011). The effects of investor sentiment on speculative trading and prices of stock and index options, Working Paper, University of Utah .

Maheu, J. M. and McCurdy, T. H. (2004). News arrival, jump dynamics, and volatility components for individual stock returns, Journal of Finance 59: 755-793.

Muravyev, D. (2016). Order flow and expected option returns, Journal of Finance 71: 673-708.

Neumann, M. and Skiadopoulos, G. (2013). Predictable dynamics in higher order risk-neutral moments: Evidence from the S\&P 500 options, Journal of Financial and Quantitative Analysis 48: 947-977.

Pan, J. and Poteshman, A. (2006). The information in options volume for future stock prices, Review of Financial Studies 19: 871-908.

Rangel, J. G. (2011). Macroeconomic news, announcements, and stock market jump intensity dynamics, Journal of Banking and Finance 35: 1263-1276. 
Santa-Clara, P. and Saretto, A. (2009). Option strategies: Good deals and margin calls, Journal of Financial Markets 12: 391-417.

Shefrin, H. M. (2001). On kernels and sentiment, Working Paper, Santa Clara University .

Sheikh, A. M. and Ronn, E. I. (1994). A characterization of the daily and intraday behavior of returns on options, Journal of Finance 49: 557-579.

Skiadopoulos, G., Hodges, S. and Clewlow, L. (1999). The dynamics of the S\&P 500 implied volatility surface, Review of Derivatives Research 3: 263-282.

Skiadopoulos, G. S. (2001). Volatility smile consistent option models: A survey, International Journal of Theoretical and Applied Finance 4: 403-437.

Taylor, S. J., Tzeng, C.-F. and Widdicks, M. (2013). Information about price and volatility jumps inferred from option prices, Journal of Futures Markets 38: 1206-1226.

Vijh, A. M. (1990). Liquidity of the CBOE equity options, Journal of Finance 45: 1157-01179. 


\section{Tables}

Table 1: Option Categories

Entries report the definition of the option delta categories in terms of their Black (1976) options delta (Panel A) and of the option expiration categories in terms of their number of days to expiration (Panel B).

\begin{tabular}{llc}
\hline Category & Name & $\begin{array}{c}\text { Delta Interval/ } \\
\text { Time to Expiration } T \text { (in days) }\end{array}$ \\
Panel $\boldsymbol{A}$ : $\boldsymbol{\text { Delta } \text { Categories }}$ & \\
1 & Deep out-of-the-money (DOTM) put & $-0.125<\Delta \leq-0.02$ \\
2 & Out-of-the-money (OTM) put & $-0.375<\Delta \leq-0.125$ \\
3 & At-the-money (ATM) put & $-0.625<\Delta \leq-0.375$ \\
4 & At-the-money (ATM) call & $0.375<\Delta \leq 0.625$ \\
5 & Out-of-the-money (OTM) call & $0.125<\Delta \leq 0.375$ \\
6 & Deep out-of-the-money (DOTM) call & $0.02<\Delta \leq 0.125$ \\
& & \\
Panel $\boldsymbol{B}:$ Maturity Categories & \\
1 & Short-term options & $10 \leq T \leq 40$ \\
2 & Medium-term options & $40<T \leq 70$ \\
3 & Long-term options & $70<T \leq 100$ \\
\hline
\end{tabular}




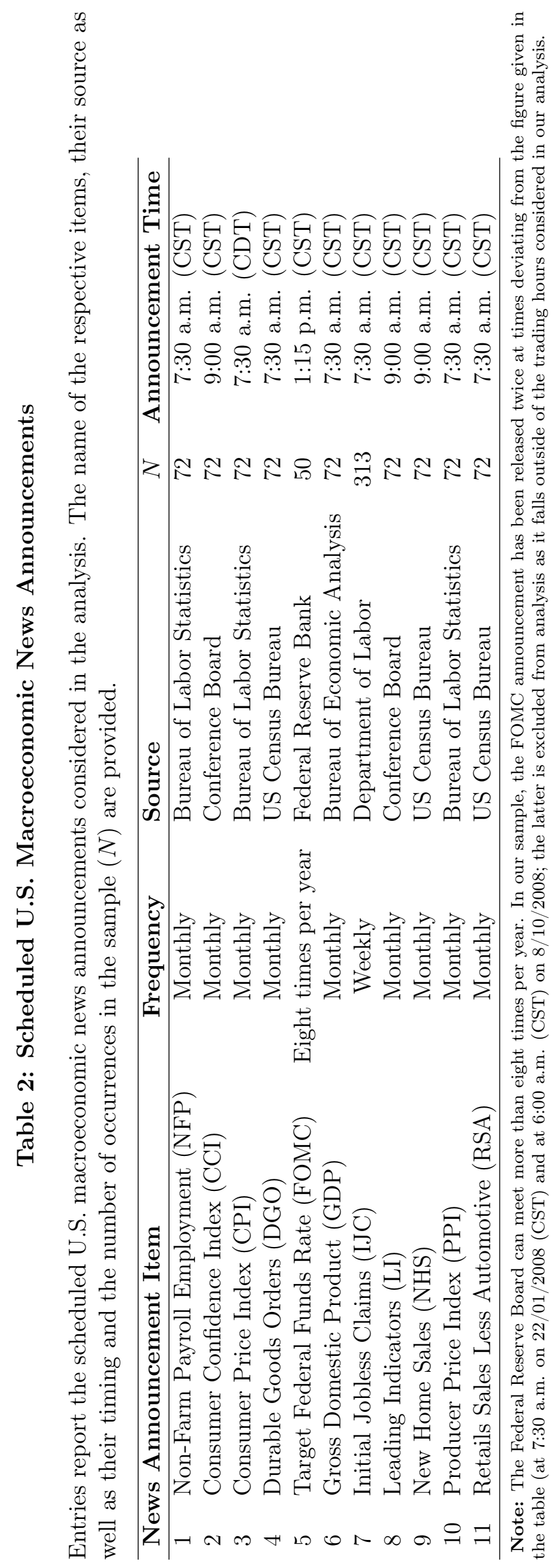




\section{Table 3: Summary Statistics of Detected Jumps}

Entries report summary statistics for the detected jumps in 10-minute returns on E-mini options for any given moneyness and maturity bucket. The number of 10-minute return observations, days, detected jumps, jump days (days with at least one jump), and the probability of a jump day to occur $P($ Jump Day), the probability of a jump to occur $P(J u m p)$, the average size for positive jumps, the average size of negative jumps and the number of negative jumps as a fraction of all jumps are reported. We use the Lee and Mykland (2008) jump detection methodology based on a significance level $\alpha=0.1 \%$ to detect jumps. The sample period is 1 January 2005 to 31 December 2010.

\begin{tabular}{ccccccc}
\hline DOTM & OTM & ATM & ATM & OTM & DOTM & Futures \\
Puts & Puts & Puts & Calls & Calls & Calls & \\
\hline
\end{tabular}

\section{Short-Term Options}

$\begin{array}{lccccccc}\text { \# Observations } & 62,142 & 63,142 & 62,734 & 62,815 & 63,074 & 61,549 & 64,665 \\ \text { \# Days } & 1,437 & 1,437 & 1,437 & 1,437 & 1,437 & 1,437 & 1437 \\ \text { \# Jumps } & 289 & 169 & 171 & 200 & 139 & 297 & 95 \\ \text { \# Jump Days } & 231 & 134 & 128 & 161 & 111 & 246 & 72 \\ \text { P(Jump Day) } & 16.08 \% & 9.32 \% & 8.91 \% & 11.20 \% & 7.72 \% & 17.12 \% & 5.01 \% \\ \text { P(Jump) } & 0.47 \% & 0.27 \% & 0.27 \% & 0.32 \% & 0.22 \% & 0.48 \% & 0.15 \% \\ \text { Avg. Jump Size } & -23.84 \% & -25.35 \% & -14.85 \% & -16.12 \% & -28.15 \% & -63.35 \% & -0.07 \% \\ \text { Avg. Positive Jump Size } & 61.79 \% & 43.80 \% & 33.76 \% & 37.03 \% & 46.85 \% & 71.59 \% & 0.85 \% \\ \text { Avg. Negative Jump Size } & -86.40 \% & -55.24 \% & -27.81 \% & -29.41 \% & -77.26 \% & -115.68 \% & -0.83 \% \\ \text { \% Negative Jumps } & 57.79 \% & 69.82 \% & 78.95 \% & 80.00 \% & 60.43 \% & 72.05 \% & 54.74 \%\end{array}$

\section{Medium-Term Options}

\begin{tabular}{lccccccc} 
\# Observations & 60,814 & 62,234 & 61,958 & 62,062 & 62,124 & 60,540 & 64,755 \\
\# Days & 1,439 & 1,439 & 1,439 & 1,439 & 1,439 & 1,439 & 1439 \\
\# Jumps & 228 & 129 & 159 & 106 & 150 & 263 & 92 \\
\# Jump Days & 181 & 100 & 111 & 80 & 125 & 218 & 70 \\
P(Jump Day) & $12.58 \%$ & $6.95 \%$ & $7.71 \%$ & $5.56 \%$ & $8.69 \%$ & $15.15 \%$ & $4.86 \%$ \\
P(Jump) & $0.37 \%$ & $0.21 \%$ & $0.26 \%$ & $0.17 \%$ & $0.24 \%$ & $0.43 \%$ & $0.14 \%$ \\
Avg. Jump Size & $-5.93 \%$ & $-7.80 \%$ & $-5.06 \%$ & $-12.97 \%$ & $-7.35 \%$ & $-29.60 \%$ & $-0.07 \%$ \\
Avg. Positive Jump Size & $34.40 \%$ & $29.28 \%$ & $26.09 \%$ & $24.49 \%$ & $34.78 \%$ & $49.32 \%$ & $0.84 \%$ \\
Avg. Negative Jump Size & $-54.88 \%$ & $-34.50 \%$ & $-14.50 \%$ & $-19.63 \%$ & $-44.22 \%$ & $-80.78 \%$ & $-0.84 \%$ \\
\% Negative Jumps & $45.18 \%$ & $58.14 \%$ & $76.73 \%$ & $84.91 \%$ & $53.33 \%$ & $60.84 \%$ & $55.43 \%$ \\
& & & & & & & \\
Long-Term Options & & & & & & & \\
\# Observations & 52,770 & 54,088 & 53,716 & 53,902 & 54,034 & 52,627 & 56,970 \\
\# Days & 1,265 & 1,265 & 1,265 & 1,265 & 1,265 & 1,265 & \\
\# Jumps & 223 & 106 & 145 & 72 & 118 & 296 & 86 \\
\# Jump Days & 159 & 80 & 112 & 62 & 97 & 222 & 67 \\
P(Jump Day) & $12.57 \%$ & $6.32 \%$ & $8.85 \%$ & $4.90 \%$ & $7.66 \%$ & $17.54 \%$ & $5.29 \%$ \\
P(Jump) & $0.42 \%$ & $0.20 \%$ & $0.27 \%$ & $0.13 \%$ & $0.22 \%$ & $0.56 \%$ & $0.15 \%$ \\
Average Jump Size & $-8.95 \%$ & $-9.78 \%$ & $-5.98 \%$ & $-10.24 \%$ & $-27.73 \%$ & $-21.76 \%$ & $-0.08 \%$ \\
Avg. Positive Jump Size & $28.38 \%$ & $19.72 \%$ & $20.47 \%$ & $16.08 \%$ & $30.14 \%$ & $41.96 \%$ & $0.87 \%$ \\
Avg. Negative Jump Size & $-37.69 \%$ & $-33.28 \%$ & $-10.46 \%$ & $-15.50 \%$ & $-70.28 \%$ & $-62.82 \%$ & $-0.82 \%$ \\
\% Negative Jumps & $56.50 \%$ & $55.66 \%$ & $85.52 \%$ & $83.33 \%$ & $57.63 \%$ & $60.81 \%$ & $55.81 \%$ \\
\hline
\end{tabular}




\section{Table 4: Summary Statistics of Quoted Sizes}

Entries report the average bid and ask trade sizes at the best bid and ask prices, respectively, for any given moneyness and maturity category. Figures are reported separately for all observations and the jump-related observations. Jumps have been detected using the Lee and Mykland (2008) jump detection methodology based on a significance level $\alpha=0.1 \%$. The average trade size is also reported. The sample spans 1 January 2005 to 31 December 2010.

\begin{tabular}{|c|c|c|c|c|c|c|}
\hline & $\begin{array}{c}\text { DOTM } \\
\text { Puts }\end{array}$ & $\begin{array}{l}\text { OTM } \\
\text { Puts }\end{array}$ & $\begin{array}{l}\text { ATM } \\
\text { Puts }\end{array}$ & $\begin{array}{l}\text { ATM } \\
\text { Calls }\end{array}$ & $\begin{array}{l}\text { OTM } \\
\text { Calls }\end{array}$ & $\begin{array}{c}\text { DOTM } \\
\text { Calls }\end{array}$ \\
\hline \multicolumn{7}{|c|}{ Panel A: Short-Term } \\
\hline \multicolumn{7}{|l|}{ Unconditional } \\
\hline Avg. Bid Size & 240.10 & 451.58 & 393.33 & 388.44 & 504.65 & 255.87 \\
\hline Avg. Ask Size & 226.79 & 437.54 & 364.30 & 355.92 & 462.23 & 233.12 \\
\hline Avg. Trading Volume & 98.22 & 105.1296 & 34.26 & 53.75 & 88.75 & 57.40 \\
\hline \multicolumn{7}{|l|}{ Conditional on Jump } \\
\hline Avg. Bid Size & 90.69 & 172.15 & 220.94 & 233.18 & 171.01 & 137.14 \\
\hline Avg. Ask Size & 140.59 & 161.22 & 199.85 & 189.87 & 161.79 & 162.55 \\
\hline Avg. Trading Volume & 82.93 & 123.59 & 61.37 & 38.35 & 79.53 & 58.77 \\
\hline \multicolumn{7}{|c|}{ Panel B: Medium-Term } \\
\hline Avg. Bid Size & 293.08 & 484.19 & 377.78 & 375.43 & 477.77 & 266.47 \\
\hline Avg. Ask Size & 268.22 & 420.91 & 335.25 & 354.28 & 415.48 & 217.79 \\
\hline Avg. Trading Volume & 30.41 & 35.83 & 9.35 & 10.85 & 27.53 & 17.68 \\
\hline \multicolumn{7}{|l|}{ Conditional on Jump } \\
\hline Avg. Bid Size & 159.13 & 223.07 & 245.57 & 273.67 & 178.37 & 144.79 \\
\hline Avg. Ask Size & 175.27 & 201.45 & 214.27 & 204.17 & 158.28 & 159.60 \\
\hline Avg. Trading Volume & 33.78 & 73.40 & 23.83 & 23.66 & 24.20 & 25.41 \\
\hline \multicolumn{7}{|l|}{ Panel C: Long-Term } \\
\hline \multicolumn{7}{|l|}{ Unconditional } \\
\hline Avg. Bid Size & 351.33 & 417.40 & 347.47 & 336.48 & 420.45 & 270.39 \\
\hline Avg. Ask Size & 306.99 & 359.16 & 305.73 & 312.79 & 347.60 & 234.67 \\
\hline Avg. Trading Volume & 11.29 & 15.04 & 4.39 & 5.00 & 10.17 & 6.86 \\
\hline \multicolumn{7}{|l|}{ Conditional on Jump } \\
\hline Avg. Bid Size & 158.91 & 245.07 & 266.19 & 241.86 & 180.94 & 154.00 \\
\hline Avg. Ask Size & 174.88 & 198.63 & 202.72 & 222.64 & 153.53 & 146.14 \\
\hline Avg. Trading Volume & 14.62 & 59.42 & 17.46 & 3.47 & 22.17 & 4.96 \\
\hline
\end{tabular}


Table 5: Relation between Jumps and Scheduled Announcements

\begin{tabular}{ccccccc}
\hline DOTM & OTM & ATM & ATM & OTM & DOTM & Futures \\
Puts & Puts & Puts & Calls & Calls & Calls & \\
\hline
\end{tabular}

Panel A: Aggregated over all News Items

Short-Term Options

\# Jumps within

10 mins of News

$P($ News $\mid$ Jump $)$

60

$20.76 \%$

42

40

57

39

61

23

P(Jump $\mid$ News $)$

$6.75 \% \quad 4.72 \% \quad 4.50 \%$

$28.50 \% \quad 28.06 \%$

$20.54 \%$

$24.21 \%$

.

Medium-Term Options

\# Jumps within

10 mins of News

$P($ News $\mid$ Jump $)$

34

$14.91 \%$

35

25

19

35

43

25

P(Jump $\mid$ News $)$

$3.82 \%$

$27.13 \% \quad 15.72 \%$

$17.92 \% \quad 23.33 \%$

$16.35 \%$

$27.17 \%$

$3.94 \% \quad 2.81 \%$

$2.14 \%$

$3.94 \%$

$4.84 \%$

$2.81 \%$

\section{Long-Term Options}

\# Jumps within

10 mins of News

$P($ News $\mid$ Jump $)$ 
Table 5: Relation between Jumps and Scheduled Announcements

Continued from previous page

\begin{tabular}{|c|c|c|c|c|c|c|c|}
\hline & $\begin{array}{c}\text { DOTM } \\
\text { Puts }\end{array}$ & $\begin{array}{l}\text { OTM } \\
\text { Puts }\end{array}$ & $\begin{array}{l}\text { ATM } \\
\text { Puts }\end{array}$ & $\begin{array}{l}\text { ATM } \\
\text { Calls }\end{array}$ & $\begin{array}{l}\text { OTM } \\
\text { Calls }\end{array}$ & $\begin{array}{c}\text { DOTM } \\
\text { Calls }\end{array}$ & Futures \\
\hline \multicolumn{8}{|c|}{ Panel B: $P($ News $\mid$ Jump $)$ Disaggregated by News Items } \\
\hline \multicolumn{8}{|c|}{ Short-Term } \\
\hline NFP & $5.19 \%$ & $8.88 \%$ & $6.43 \%$ & $6.00 \%$ & $5.04 \%$ & $4.38 \%$ & $11.58 \%$ \\
\hline CCI & $0.35 \%$ & $0.00 \%$ & $1.75 \%$ & $1.50 \%$ & $0.00 \%$ & $1.35 \%$ & $0.00 \%$ \\
\hline CPI & $2.42 \%$ & $2.37 \%$ & $2.92 \%$ & $3.50 \%$ & $4.32 \%$ & $0.67 \%$ & $0.00 \%$ \\
\hline DGO & $2.08 \%$ & $0.59 \%$ & $2.92 \%$ & $2.00 \%$ & $1.44 \%$ & $2.02 \%$ & $0.00 \%$ \\
\hline FOMC & $0.69 \%$ & $1.18 \%$ & $2.34 \%$ & $1.50 \%$ & $2.16 \%$ & $0.67 \%$ & $9.47 \%$ \\
\hline GDP & $2.08 \%$ & $1.78 \%$ & $1.75 \%$ & $3.50 \%$ & $2.88 \%$ & $2.69 \%$ & $1.05 \%$ \\
\hline IJC & $7.96 \%$ & $5.92 \%$ & $7.60 \%$ & $9.50 \%$ & $11.51 \%$ & $8.75 \%$ & $1.05 \%$ \\
\hline LI & $0.35 \%$ & $0.00 \%$ & $0.58 \%$ & $0.50 \%$ & $0.00 \%$ & $0.34 \%$ & $0.00 \%$ \\
\hline NHS & $0.35 \%$ & $0.59 \%$ & $0.00 \%$ & $1.00 \%$ & $0.00 \%$ & $0.67 \%$ & $1.05 \%$ \\
\hline PPI & $1.73 \%$ & $4.14 \%$ & $0.00 \%$ & $1.50 \%$ & $1.44 \%$ & $1.68 \%$ & $0.00 \%$ \\
\hline RSA & $1.04 \%$ & $3.55 \%$ & $0.00 \%$ & $1.00 \%$ & $2.88 \%$ & $1.01 \%$ & $0.00 \%$ \\
\hline \multicolumn{8}{|c|}{ Medium-Term } \\
\hline NFP & $6.14 \%$ & $8.53 \%$ & $5.66 \%$ & $5.66 \%$ & $10.00 \%$ & $2.28 \%$ & $14.13 \%$ \\
\hline CCI & $0.00 \%$ & $0.78 \%$ & $1.89 \%$ & $1.89 \%$ & $0.67 \%$ & $0.38 \%$ & $0.00 \%$ \\
\hline CPI & $0.88 \%$ & $0.78 \%$ & $1.89 \%$ & $0.94 \%$ & $1.33 \%$ & $2.28 \%$ & $0.00 \%$ \\
\hline DGO & $0.44 \%$ & $1.55 \%$ & $0.63 \%$ & $0.00 \%$ & $2.67 \%$ & $1.52 \%$ & $0.00 \%$ \\
\hline FOMC & $1.75 \%$ & $3.10 \%$ & $2.52 \%$ & $1.89 \%$ & $1.33 \%$ & $0.76 \%$ & $9.78 \%$ \\
\hline GDP & $0.44 \%$ & $3.88 \%$ & $0.00 \%$ & $1.89 \%$ & $1.33 \%$ & $0.76 \%$ & $1.09 \%$ \\
\hline IJC & $3.07 \%$ & $10.08 \%$ & $1.89 \%$ & $4.72 \%$ & $5.33 \%$ & $6.08 \%$ & $1.09 \%$ \\
\hline LI & $0.44 \%$ & $0.78 \%$ & $0.00 \%$ & $0.00 \%$ & $0.67 \%$ & $1.14 \%$ & $0.00 \%$ \\
\hline NHS & $0.00 \%$ & $0.00 \%$ & $0.63 \%$ & $1.89 \%$ & $0.67 \%$ & $0.76 \%$ & $1.09 \%$ \\
\hline PPI & $1.32 \%$ & $0.78 \%$ & $0.63 \%$ & $0.00 \%$ & $0.00 \%$ & $0.76 \%$ & $0.00 \%$ \\
\hline RSA & $0.44 \%$ & $1.55 \%$ & $0.00 \%$ & $0.94 \%$ & $0.67 \%$ & $0.76 \%$ & $0.00 \%$ \\
\hline \multicolumn{8}{|c|}{ Long-Term } \\
\hline NFP & $3.14 \%$ & $6.60 \%$ & $4.14 \%$ & $5.56 \%$ & $7.63 \%$ & $3.04 \%$ & $10.47 \%$ \\
\hline CCI & $1.35 \%$ & $1.89 \%$ & $2.07 \%$ & $0.00 \%$ & $3.39 \%$ & $0.00 \%$ & $2.33 \%$ \\
\hline CPI & $0.90 \%$ & $0.00 \%$ & $1.38 \%$ & $0.00 \%$ & $5.93 \%$ & $1.01 \%$ & $0.00 \%$ \\
\hline DGO & $2.69 \%$ & $0.00 \%$ & $0.69 \%$ & $0.00 \%$ & $0.85 \%$ & $1.69 \%$ & $0.00 \%$ \\
\hline FOMC & $0.45 \%$ & $3.77 \%$ & $3.45 \%$ & $1.39 \%$ & $1.69 \%$ & $1.35 \%$ & $10.47 \%$ \\
\hline GDP & $0.90 \%$ & $0.94 \%$ & $0.00 \%$ & $1.39 \%$ & $3.39 \%$ & $1.69 \%$ & $1.16 \%$ \\
\hline IJC & $4.04 \%$ & $5.66 \%$ & $0.69 \%$ & $2.78 \%$ & $8.47 \%$ & $3.72 \%$ & $1.16 \%$ \\
\hline LI & $1.35 \%$ & $0.00 \%$ & $0.00 \%$ & $0.00 \%$ & $0.00 \%$ & $0.68 \%$ & $0.00 \%$ \\
\hline NHS & $0.90 \%$ & $0.94 \%$ & $2.07 \%$ & $1.39 \%$ & $0.00 \%$ & $0.34 \%$ & $1.16 \%$ \\
\hline PPI & $0.45 \%$ & $0.94 \%$ & $0.00 \%$ & $0.00 \%$ & $0.85 \%$ & $1.35 \%$ & $0.00 \%$ \\
\hline RSA & $0.45 \%$ & $0.94 \%$ & $0.00 \%$ & $5.56 \%$ & $0.00 \%$ & $2.36 \%$ & $0.00 \%$ \\
\hline
\end{tabular}

Continued on next page 
Table 5: Relation between Jumps and Scheduled Announcements

Continued from previous page

\begin{tabular}{ccccccc}
\hline DOTM & OTM & ATM & ATM & OTM & DOTM & Futures \\
Puts & Puts & Puts & Calls & Calls & Calls & \\
\hline
\end{tabular}

Panel C: P(Jump|News) Disaggregated by News Items

Short-Term

\begin{tabular}{lccccccc} 
NFP & $20.83 \%$ & $20.83 \%$ & $15.28 \%$ & $16.67 \%$ & $9.72 \%$ & $18.06 \%$ & $15.28 \%$ \\
CCI & $1.39 \%$ & $0.00 \%$ & $4.17 \%$ & $4.17 \%$ & $0.00 \%$ & $5.56 \%$ & $0.00 \%$ \\
CPI & $9.72 \%$ & $5.56 \%$ & $6.94 \%$ & $9.72 \%$ & $8.33 \%$ & $2.78 \%$ & $0.00 \%$ \\
DGO & $8.33 \%$ & $1.39 \%$ & $6.94 \%$ & $5.56 \%$ & $2.78 \%$ & $8.33 \%$ & $0.00 \%$ \\
FOMC & $4.00 \%$ & $4.00 \%$ & $8.00 \%$ & $6.00 \%$ & $6.00 \%$ & $4.00 \%$ & $18.00 \%$ \\
GDP & $8.33 \%$ & $4.17 \%$ & $4.17 \%$ & $9.72 \%$ & $5.56 \%$ & $11.11 \%$ & $1.39 \%$ \\
IJC & $7.35 \%$ & $3.19 \%$ & $4.15 \%$ & $6.07 \%$ & $5.11 \%$ & $8.31 \%$ & $0.32 \%$ \\
LI & $1.39 \%$ & $0.00 \%$ & $1.39 \%$ & $1.39 \%$ & $0.00 \%$ & $1.39 \%$ & $0.00 \%$ \\
NHS & $1.39 \%$ & $1.39 \%$ & $0.00 \%$ & $2.78 \%$ & $0.00 \%$ & $2.78 \%$ & $1.39 \%$ \\
PPI & $6.94 \%$ & $9.72 \%$ & $0.00 \%$ & $4.17 \%$ & $2.78 \%$ & $6.94 \%$ & $0.00 \%$ \\
RSA & $4.17 \%$ & $8.33 \%$ & $0.00 \%$ & $2.78 \%$ & $5.56 \%$ & $4.17 \%$ & $0.00 \%$ \\
& & & & & & & \\
Medium-Term & & & & & & & \\
NFP & $19.44 \%$ & $15.28 \%$ & $12.50 \%$ & $8.33 \%$ & $20.83 \%$ & $8.33 \%$ & $18.06 \%$ \\
CCI & $0.00 \%$ & $1.39 \%$ & $4.17 \%$ & $2.78 \%$ & $1.39 \%$ & $1.39 \%$ & $0.00 \%$ \\
CPI & $2.78 \%$ & $1.39 \%$ & $4.17 \%$ & $1.39 \%$ & $2.78 \%$ & $8.33 \%$ & $0.00 \%$ \\
DGO & $1.39 \%$ & $2.78 \%$ & $1.39 \%$ & $0.00 \%$ & $5.56 \%$ & $5.56 \%$ & $0.00 \%$ \\
FOMC & $8.00 \%$ & $8.00 \%$ & $8.00 \%$ & $4.00 \%$ & $4.00 \%$ & $4.00 \%$ & $18.00 \%$ \\
GDP & $1.39 \%$ & $6.94 \%$ & $0.00 \%$ & $2.78 \%$ & $2.78 \%$ & $2.78 \%$ & $1.39 \%$ \\
IJC & $2.24 \%$ & $4.15 \%$ & $0.96 \%$ & $1.60 \%$ & $2.56 \%$ & $5.11 \%$ & $0.32 \%$ \\
LI & $1.39 \%$ & $1.39 \%$ & $0.00 \%$ & $0.00 \%$ & $1.39 \%$ & $4.17 \%$ & $0.00 \%$ \\
NHS & $0.00 \%$ & $0.00 \%$ & $1.39 \%$ & $2.78 \%$ & $1.39 \%$ & $2.78 \%$ & $1.39 \%$ \\
PPI & $4.17 \%$ & $1.39 \%$ & $1.39 \%$ & $0.00 \%$ & $0.00 \%$ & $2.78 \%$ & $0.00 \%$ \\
RSA & $1.39 \%$ & $2.78 \%$ & $0.00 \%$ & $1.39 \%$ & $1.39 \%$ & $2.78 \%$ & $0.00 \%$ \\
Long-Term & & & & & & & \\
NFP & & & & & & & \\
CCI & $9.72 \%$ & $9.72 \%$ & $8.33 \%$ & $5.56 \%$ & $12.50 \%$ & $12.50 \%$ & $12.50 \%$ \\
CPI & $4.17 \%$ & $2.78 \%$ & $4.17 \%$ & $0.00 \%$ & $5.56 \%$ & $0.00 \%$ & $2.78 \%$ \\
DGO & $2.78 \%$ & $0.00 \%$ & $2.78 \%$ & $0.00 \%$ & $9.72 \%$ & $4.17 \%$ & $0.00 \%$ \\
FOMC & $8.33 \%$ & $0.00 \%$ & $1.39 \%$ & $0.00 \%$ & $1.39 \%$ & $6.94 \%$ & $0.00 \%$ \\
GDP & $2.00 \%$ & $8.00 \%$ & $10.00 \%$ & $2.00 \%$ & $4.00 \%$ & $8.00 \%$ & $18.00 \%$ \\
IJC & $2.78 \%$ & $1.39 \%$ & $0.00 \%$ & $1.39 \%$ & $5.56 \%$ & $6.94 \%$ & $1.39 \%$ \\
LI & $2.88 \%$ & $1.92 \%$ & $0.32 \%$ & $0.64 \%$ & $3.19 \%$ & $3.51 \%$ & $0.32 \%$ \\
NHS & $4.17 \%$ & $0.00 \%$ & $0.00 \%$ & $0.00 \%$ & $0.00 \%$ & $2.78 \%$ & $0.00 \%$ \\
PPI & $2.78 \%$ & $1.39 \%$ & $4.17 \%$ & $1.39 \%$ & $0.00 \%$ & $1.39 \%$ & $1.39 \%$ \\
RSA & $1.39 \%$ & $1.39 \%$ & $0.00 \%$ & $0.00 \%$ & $1.39 \%$ & $5.56 \%$ & $0.00 \%$ \\
SI & $1.39 \%$ & $1.39 \%$ & $0.00 \%$ & $5.56 \%$ & $0.00 \%$ & $9.72 \%$ & $0.00 \%$ \\
\hline
\end{tabular}

Entries report summary statistics on the relation between detected jumps and scheduled macroeconomic news announcement items for all investigated moneyness and maturity categories. The probability of a jump being related to a specific news announcement $P(N e w s \mid J u m p)$ and the probability of a news announcement leading to a jump $P(J u m p \mid N e w s)$ are reported. Panel A reports these statistics aggregated over all considered news announcement items and Panel $\mathrm{B}$ and $\mathrm{C}$ report them disaggregated by individual announcement items. Jumps have been detected using the Lee and Mykland (2008) jump detection methodology based on a significance level $\alpha=0.1 \%$. A jump is defined to be related to news if it occurred within \pm 10 minutes of a scheduled news announcement 
Table 6: Information Shocks and Illiquidity as Jump Determinants

\begin{tabular}{|c|c|c|c|}
\hline & Short-Term & Medium-Term & Long-Term \\
\hline \multicolumn{4}{|c|}{ Panel A: News Covariates } \\
\hline$c$ & $-3.8579 * * *$ & $-4.1575 * * *$ & $-4.8081 * * *$ \\
\hline$N F P_{t}$ & $0.7506^{* * *}$ & $0.6315^{* *}$ & -0.0617 \\
\hline$C C I_{t}$ & -1.0005 & -0.6084 & -0.0903 \\
\hline$C P I_{t}$ & 0.2371 & -0.2751 & 0.2545 \\
\hline$D G O_{t}$ & 0.1317 & -0.9814 & -1.1075 \\
\hline$F O M C_{t}$ & - & - & - \\
\hline$G D P_{t}$ & 0.1568 & -0.1131 & $0.7241^{* * *}$ \\
\hline$I J C_{t}$ & $0.287^{*}$ & -0.0701 & 0.1289 \\
\hline$L I_{t}$ & -0.9801 & -0.6891 & - \\
\hline$N H S_{t}$ & - & -6.6603 & - \\
\hline$P P I_{t}$ & -0.0452 & -0.137 & -0.0892 \\
\hline$R S A_{S} t$ & -0.1407 & -1.0811 & $0.7789^{* *}$ \\
\hline \multicolumn{4}{|c|}{ Panel B: News and Liquidity Covariates } \\
\hline$c$ & $-3.4309 * * *$ & $-3.7201^{* * *}$ & $-4.1162^{* * *}$ \\
\hline$s B A_{t-1}$ & $0.1161^{* * *}$ & $0.1431^{* * *}$ & $0.1737^{* * *}$ \\
\hline BidSize $_{t-1}$ & $-0.0026^{* *}$ & -0.002 & -0.0025 \\
\hline AskSize $e_{t-1}$ & -0.0015 & -0.0018 & -0.0035 \\
\hline$N F P_{t}$ & $0.5904^{* * *}$ & $0.5042^{* *}$ & -0.2296 \\
\hline$C C I_{t}$ & -0.4622 & -0.2161 & 0.2941 \\
\hline$C P I_{t}$ & 0.1338 & -0.3598 & 0.0227 \\
\hline$D G O_{t}$ & 0.0592 & -1.0874 & -1.3451 \\
\hline$F O M C_{t}$ & - & - & - \\
\hline$G D P_{t}$ & -0.0079 & -0.3073 & $0.6001^{* *}$ \\
\hline$I J C_{t}$ & 0.1776 & -0.2565 & -0.1036 \\
\hline$L I_{t}$ & -0.4828 & -0.3253 & - \\
\hline$N H S_{t}$ & - & -4.9552 & - \\
\hline$P P I_{t}$ & -0.1628 & -0.2543 & -0.3161 \\
\hline$R S A_{t}$ & -0.2575 & -1.0971 & 0.4082 \\
\hline
\end{tabular}

Entries report the estimation results for the logistic regression models in equations (8) and (9) (Panel $\mathrm{A}$ and $\mathrm{B}$, respectively). The estimation is performed separately for short, medium, and long-term options on a sample pooled across all delta categories. Only news-related observations are considered. The estimation is performed via Maximum Likelihood and $* * *, * *, *$ report statistical significance on a $1 \%, 5 \%$, and $10 \%$ significance level, respectively. The sample period is 1 January 2005 to 31 December 2010 . 
Table 7: Information Shocks, Volume, and Illiquidity as Jump Determinants

\begin{tabular}{|c|c|c|c|}
\hline & Short Term & Medium Term & Long Term \\
\hline \multicolumn{4}{|c|}{ Panel A: News, Volume, and Liquidity Covariates } \\
\hline$c$ & $-3.4042^{* * *}$ & $-3.6822^{* * *}$ & $-4.0221^{* * *}$ \\
\hline$s B A_{t-1}$ & $0.1142^{* * *}$ & $0.1463^{* * *}$ & $0.1703^{* * *}$ \\
\hline BidSize $_{t-1}$ & $-0.0025^{* *}$ & -0.0021 & -0.0026 \\
\hline AskSize $_{t-1}$ & -0.0014 & -0.0019 & -0.0037 \\
\hline Volume $_{t-1, t}$ & -0.002 & 0.0024 & 0.0011 \\
\hline$N F P_{t}$ & $0.5879^{* * *}$ & $0.4857^{*}$ & -0.2329 \\
\hline$C C I_{t}$ & -0.3124 & -0.3031 & 0.2957 \\
\hline$C P I_{t}$ & 0.1126 & -0.3878 & 0.0331 \\
\hline$D G O_{t}$ & 0.0448 & -1.1291 & -1.4186 \\
\hline$F O M C_{t}$ & - & - & - \\
\hline$G D P_{t}$ & -0.0156 & -0.3327 & $0.5768^{* *}$ \\
\hline$I J C_{t}$ & 0.1623 & -0.2235 & -0.1085 \\
\hline$L I_{t}$ & -0.3449 & -0.3942 & - \\
\hline$N H S_{t}$ & - & -5.4217 & - \\
\hline$P P I_{t}$ & -0.1783 & -0.28 & -0.304 \\
\hline$R S A_{t}$ & -0.2734 & -1.1052 & 0.4241 \\
\hline
\end{tabular}

Panel B: Volume and Liquidity Covariates for no-news related jumps

$\begin{array}{lccc}c & -5.2846^{* * *} & -5.3888^{* * *} & -5.1133^{* * *} \\ \text { sBA }_{t-1} & 0.3338^{* * *} & 0.292^{* * *} & 0.2353^{* * *} \\ \text { BidSize }_{t-1} & -0.0028^{* * *} & -0.0019^{* * *} & -0.0023^{* * *} \\ \text { AskSize }_{t-1} & -0.0022^{* * *} & -0.0024^{* * *} & -0.0025^{* * *} \\ {\text { V } \text { olume }_{t-1, t}}^{* *} & 0.0006^{* * *} & 0.0007^{* * *} & 0.0013^{* * *}\end{array}$

Entries report the estimation results for the logistic regression models in equations (11) and (12) (Panel A and B, respectively). The estimation is performed separately for short, medium, and longterm options on a sample pooled across all delta categories. Panel A considers only news-related observations and Panel B considers only non-news-related observations. The estimation is performed via Maximum Likelihood and $* * *, * *, *$ report statistical significance on a $1 \%, 5 \%$, and $10 \%$ significance level, respectively. The sample period is 1 January 2005 to 31 December 2010. 
Table 8: Summary Statistics of Detected Jumps (Non-Crisis and Crisis Subsample)

\begin{tabular}{ccccccc}
\hline DOTM & OTM & ATM & ATM & OTM & DOTM & Futures \\
Puts & Puts & Puts & Calls & Calls & Calls & \\
\hline
\end{tabular}

Panel A: Non-Crisis Subsample

Short-Term

\# Observations

\# Jumps

25,501

149

26,419

$\begin{array}{cc}26,044 & 26,122 \\ 54 & 101 \\ 46 & 85\end{array}$

26,359

24,912

27,720

\# Jump Days

124

98

85

88

115

40

$20.13 \%$

78

$7.47 \%$

68

95

32

P(Jump Day)

$0.58 \%$

$0.37 \%$

$0.21 \%$

$13.80 \%$

$11.04 \%$

$15.42 \%$

$5.19 \%$

Avg. Jump Size

$-31.44 \%$

$-30.79 \%$

$0.39 \%$

$0.33 \%$

$0.46 \%$

$0.14 \%$

\% Negative Jumps

$59.73 \%$

$71.43 \%$

$85.19 \%$

$-21.31 \%$

$-36.98 \%$

$-35.99 \%$

$0.00 \%$

Medium-Term

\# Observations

\# Jumps

24,237

25,356

25,092

$83.17 \%$

$62.50 \%$

$66.96 \%$

$50.00 \%$

\# Jump Days

120

55

25,204

25,262

23,937

27,540

97

45

47

54

89

98

39

P(Jump Day)

$15.85 \%$

$7.35 \%$

36

44

75

$5.88 \%$

$7.19 \%$

$12.25 \%$

83

31

$0.50 \%$

$0.22 \%$

$0.19 \%$

$0.21 \%$

$0.35 \%$

$13.56 \%$

$5.07 \%$

$-5.49 \%$

$-11.71 \%$

$-14.79 \%$

$-10.78 \%$

$-4.17 \%$

$0.41 \%$

$0.14 \%$

$46.67 \%$

$65.45 \%$

$95.74 \% \quad 87.04 \%$

$47.19 \%$

$-16.26 \%$

$0.00 \%$

$50.00 \%$

$51.28 \%$

\section{Long-Term}

\# Observations

\# Jumps

17,569

18,427

18,082

18,252

18,369

37

44

17,431

20,700

77

38

43

35

36

103

36

\# Jump Days

$16.74 \%$

$6.09 \%$

$7.83 \%$

$7.63 \%$

$7.83 \%$

79

30

P(Jump)

$0.66 \%$

$0.21 \%$

$0.24 \%$

$0.20 \%$

$0.24 \%$

$17.17 \%$

$6.52 \%$

$-3.60 \%$

$-2.04 \%$

$-9.34 \%$

$-7.08 \%$

$-5.81 \%$

$0.59 \%$

$0.17 \%$

\% Negative Jumps

$48.28 \%$

$55.26 \%$

$93.02 \%$

$86.49 \%$

$61.36 \%$

$-21.31 \%$

$-0.02 \%$

$53.40 \%$

$52.78 \%$

Continued on next page 
Table 8: Summary Statistics of Detected Jumps (Non-Crisis and Crisis Subsample) Continued from previous page

\begin{tabular}{ccccccc}
\hline DOTM & OTM & ATM & ATM & OTM & DOTM & Futures \\
Puts & Puts & Puts & Calls & Calls & Calls & \\
\hline
\end{tabular}

Panel B: Crisis Subsample

\section{Short-Term}

\# Observations

\# Jumps

\# Jump Days

$P($ Jump Day $)$

$P($ Jump $)$

Avg. Jump Size

\% Negative Jumps

$\begin{array}{ccc}36,596 & 36,678 & 36,645 \\ 140 & 71 & 117 \\ 107 & 56 & 82 \\ 13.05 \% & 6.83 \% & 10.00 \% \\ 0.38 \% & 0.19 \% & 0.32 \% \\ -32.31 \% & -30.03 \% & -17.59 \% \\ 60.71 \% & 71.83 \% & 82.91 \%\end{array}$

36,648
99
76
$9.27 \%$
$0.27 \%$
$-21.40 \%$
$82.83 \%$

36,670

36,592

36,900

182

55

43

151

40

$5.24 \% \quad 18.41 \% \quad 4.88 \%$

$\begin{array}{lll}5.14 \% & 0.50 \% & 0.15 \%\end{array}$

$-56.32 \% \quad-50.81 \% \quad 0.06 \%$

Medium-Term

\# Observations

\# Jumps

36,5
10
83

\# Jump Days

P(Jump Day)

$P($ Jump $)$

Avg. Jump Size

$\%$ Negative Jumps

36,833

36,821
112

36,813

52

36,817

75

36

$10.05 \%$

55

75

$0.29 \%$

$6.66 \%$

$9.08 \%$

$4.36 \%$

61

50

165

37,170

$-3.13 \%$

$0.20 \%$

$0.30 \%$

$0.14 \%$

$6.05 \%$

135

53

39

$45.79 \%$

$-9.71 \%$

$-5.89 \%$

$-12.40 \%$

$0.17 \%$

$16.34 \%$

$4.72 \%$

$66.22 \%$

$80.36 \%$

$90.38 \%$

$-12.36 \%$

$0.45 \%$

$0.14 \%$

$-29.17 \%$

$0.06 \%$

$57.38 \%$

$60.61 \%$

$49.06 \%$

\section{Long-Term}

\# Observations

\# Jumps

35,156

35,616

35,590

35,606

35,620

35,151

36,225

107

68

102

35

74

193

50

\# Jump Days

82

52

76

27

61

143

37

P(Jump Day)

$10.20 \%$

$6.46 \%$

$9.44 \%$

$3.35 \%$

$7.58 \%$

$17.76 \%$

$4.60 \%$

$P$ (Jump)

$0.30 \%$

$0.19 \%$

$0.29 \%$

$0.10 \%$

$0.21 \%$

$0.55 \%$

$0.14 \%$

$-3.87 \% \quad-7.75 \%$

$-5.59 \%$

$-7.77 \%$

$-17.44 \%$

$-28.65 \%$

$0.01 \%$

$\%$ Negative Jumps

$48.60 \% \quad 61.76 \%$

$87.25 \% \quad 88.57 \%$

$54.05 \% \quad 62.69 \%$

$50.00 \%$

Entries report summary statistics for the detected jumps for all investigated moneyness and maturity categories over the non-crisis subsample (Panel A) and crisis subsample (Panel B). The number of detected jumps, the number of jump days (days with at least one jump), the probability of a jump day to occur $P($ Jump Day), the probability of a jump to occur $P(J u m p)$ and the number of negative jumps as a fraction of all jumps are reported. Jumps have been detected using the Lee and Mykland (2008) jump detection methodology based on a significance level $\alpha=0.1 \%$. The sample period is 1January 2005 to 31 July 2007 for the non-crisis subsample and 1 August 2007 to 31 December 2010 for the crisis subsample. 
Table 9: Relation between Jumps \& Scheduled Announcements (Non-Crisis \& Crisis Subsample)

\begin{tabular}{ccccccc}
\hline DOTM & OTM & ATM & ATM & OTM & DOTM & Futures \\
Puts & Puts & Puts & Calls & Calls & Calls & \\
\hline
\end{tabular}

\section{Panel A: Non-Crisis Subsample}

Short-Term

\# Jumps within

10 mins of News

$P($ News $\mid$ Jump $)$

19

$\begin{array}{cc}20 & 9 \\ 20.41 \% & 16.67 \%\end{array}$

26

18

14

11

P(Jump $\mid$ News $)$

$12.75 \%$

$5.21 \% \quad 2.34 \%$

$25.74 \%$

$20.45 \%$

$12.17 \%$

$27.50 \%$

$4.95 \%$

$6.77 \%$

$4.69 \%$

$3.65 \%$

$2.86 \%$

Medium-Term

\# Jumps within

10 mins of News

$P($ News $\mid$ Jump $)$

11

$20.00 \%$

6

5

15

10

12

P(Jump $\mid$ News $)$

$2.86 \%$

$2.86 \%$

$1.56 \%$

$1.30 \%$

$16.85 \%$

$10.20 \%$

$30.77 \%$

Long-Term

\# Jumps within

10 mins of News

P(News $\mid$ Jump $)$

9

5

7

1

9

9

12

P(Jump $\mid$ News $)$

$2.34 \%$

$13.16 \% \quad 16.28 \%$

$2.70 \%$

$20.45 \%$

$8.74 \%$

$33.33 \%$

$1.30 \%$

$1.82 \%$

$0.26 \%$

$2.34 \%$

$2.34 \%$

$3.13 \%$

Panel B: Crisis Subsample

Short-Term

\# Jumps within

10 mins of News

P(News|Jump)

41

22

31

31

21

47

12

P(Jump $\mid$ News $)$

$29.29 \% \quad 30.99 \%$

$26.50 \%$

$31.31 \%$

$41.18 \%$

$25.82 \%$

$21.82 \%$

$8.13 \%$

$4.37 \%$

$6.15 \%$

$6.15 \%$

$4.17 \%$

$9.33 \%$

$2.38 \%$

Medium-Term

\# Jumps within

10 mins of News

$P$ (News|Jump $)$

23

24

19

14

20

33

13

$P($ Jump $\mid$ News $)$

$4.56 \%$

$3.76 \% \quad 3.77 \%$

$26.92 \%$

$20.00 \%$

$24.53 \%$

$2.58 \%$

\section{Long-Term}

\# Jumps within

10 mins of News

P(News $\mid$ Jump $)$

23

16

14

11

24

35

11

P(Jump $\mid$ News $)$

$21.50 \% \quad 23.53 \% \quad 13.73 \%$

$31.43 \%$

$32.43 \%$

$18.13 \%$

$22.00 \%$

$4.56 \%$

$3.17 \% \quad 2.78 \%$

$2.18 \% \quad 4.76 \%$

$6.94 \%$

$2.18 \%$

Entries report summary statistics on the relation between detected jumps and macroeconomic news announcements for all investigated moneyness and maturity categories over the non-crisis subsample (Panel A) and crisis subsample (Panel B). The number of jumps that occurred within \pm 10 minutes of a scheduled news announcement, the probability of a news announcement leading to a jump $P($ Jump $\mid N e w s)$ as well as the probability of a jump being related to a news announcement $P($ News $\mid$ Jump $)$ are reported. Jumps have been detected using the Lee and Mykland (2008) jump detection methodology based on a significance level $\alpha=0.1 \%$. The sample period is 1 Januray 2005 to 31 July 2007 for the non-crisis and 1 August 2007 to 31 December 2010 for the crisis subsample. 
Table 10: Relation between Jumps and Scheduled Announcements Disaggregated by Announcement Items (Non-Crisis and Crisis Subsample)

\begin{tabular}{ccccccc}
\hline DOTM & OTM & ATM & ATM & OTM & DOTM & Futures \\
Puts & Puts & Puts & Calls & Calls & Calls & \\
\hline
\end{tabular}

Panel A: $P($ News $\mid$ Jump $)$ over the Non-Crisis Subsample

\section{Short-Term Options}

$\begin{array}{lccccccc}\text { NFP } & 1.34 \% & 4.08 \% & 5.56 \% & 1.98 \% & 2.27 \% & 1.74 \% & 10.00 \% \\ \text { CCI } & 0.67 \% & 0.00 \% & 1.85 \% & 0.00 \% & 0.00 \% & 2.61 \% & 0.00 \% \\ \text { CPI } & 2.68 \% & 3.06 \% & 3.70 \% & 4.95 \% & 2.27 \% & 0.87 \% & 0.00 \% \\ \text { DGO } & 2.01 \% & 0.00 \% & 1.85 \% & 0.00 \% & 2.27 \% & 0.87 \% & 0.00 \% \\ \text { FOMC } & 0.00 \% & 0.00 \% & 1.85 \% & 0.99 \% & 0.00 \% & 0.00 \% & 12.50 \% \\ \text { GDP } & 2.68 \% & 0.00 \% & 1.85 \% & 2.97 \% & 1.14 \% & 0.00 \% & 0.00 \% \\ \text { IJC } & 3.36 \% & 8.16 \% & 0.00 \% & 11.88 \% & 10.23 \% & 3.48 \% & 2.50 \% \\ \text { LI } & 0.00 \% & 0.00 \% & 0.00 \% & 0.00 \% & 0.00 \% & 0.00 \% & 0.00 \% \\ \text { NHS } & 0.00 \% & 0.00 \% & 0.00 \% & 0.99 \% & 0.00 \% & 0.00 \% & 2.50 \% \\ \text { PPI } & 0.67 \% & 6.12 \% & 0.00 \% & 1.98 \% & 2.27 \% & 2.61 \% & 0.00 \% \\ \text { RSA } & 0.00 \% & 5.10 \% & 0.00 \% & 1.98 \% & 1.14 \% & 0.87 \% & 0.00 \%\end{array}$

Medium-Term Options

$\begin{array}{lccccccc}\text { NFP } & 3.33 \% & 7.27 \% & 2.13 \% & 0.00 \% & 7.87 \% & 2.04 \% & 12.82 \% \\ \text { CCI } & 0.00 \% & 0.00 \% & 2.13 \% & 1.85 \% & 1.12 \% & 0.00 \% & 0.00 \% \\ \text { CPI } & 0.83 \% & 1.82 \% & 4.26 \% & 1.85 \% & 2.25 \% & 2.04 \% & 0.00 \% \\ \text { DGO } & 0.83 \% & 0.00 \% & 0.00 \% & 0.00 \% & 0.00 \% & 1.02 \% & 0.00 \% \\ \text { FOMC } & 0.83 \% & 1.82 \% & 2.13 \% & 0.00 \% & 0.00 \% & 1.02 \% & 12.82 \% \\ \text { GDP } & 0.83 \% & 5.45 \% & 0.00 \% & 1.85 \% & 1.12 \% & 1.02 \% & 0.00 \% \\ \text { IJC } & 0.83 \% & 3.64 \% & 2.13 \% & 1.85 \% & 3.37 \% & 0.00 \% & 2.56 \% \\ \text { LI } & 0.00 \% & 0.00 \% & 0.00 \% & 0.00 \% & 1.12 \% & 1.02 \% & 0.00 \% \\ \text { NHS } & 0.00 \% & 0.00 \% & 0.00 \% & 1.85 \% & 0.00 \% & 1.02 \% & 2.56 \% \\ \text { PPI } & 1.67 \% & 0.00 \% & 0.00 \% & 0.00 \% & 0.00 \% & 0.00 \% & 0.00 \% \\ \text { RSA } & 0.00 \% & 0.00 \% & 0.00 \% & 0.00 \% & 1.12 \% & 1.02 \% & 0.00 \%\end{array}$

\section{Long-Term Options}

\begin{tabular}{llllllll} 
NFP & $0.86 \%$ & $2.63 \%$ & $2.33 \%$ & $0.00 \%$ & $4.55 \%$ & $2.91 \%$ & $8.33 \%$ \\
CCI & $2.59 \%$ & $2.63 \%$ & $2.33 \%$ & $0.00 \%$ & $6.82 \%$ & $0.00 \%$ & $5.56 \%$ \\
CPI & $0.00 \%$ & $0.00 \%$ & $2.33 \%$ & $0.00 \%$ & $4.55 \%$ & $0.00 \%$ & $0.00 \%$ \\
DGO & $1.72 \%$ & $0.00 \%$ & $0.00 \%$ & $0.00 \%$ & $0.00 \%$ & $0.97 \%$ & $0.00 \%$ \\
FOMC & $0.00 \%$ & $5.26 \%$ & $4.65 \%$ & $0.00 \%$ & $0.00 \%$ & $2.91 \%$ & $13.89 \%$ \\
GDP & $0.00 \%$ & $0.00 \%$ & $0.00 \%$ & $0.00 \%$ & $0.00 \%$ & $0.91 \%$ & $0.00 \%$ \\
IJC & $0.86 \%$ & $0.00 \%$ & $0.00 \%$ & $0.00 \%$ & $4.55 \%$ & $0.00 \%$ & $2.78 \%$ \\
LI & $0.86 \%$ & $0.00 \%$ & $0.00 \%$ & $0.00 \%$ & $0.00 \%$ & $0.00 \%$ & $0.00 \%$ \\
NHS & $1.72 \%$ & $0.00 \%$ & $4.65 \%$ & $2.70 \%$ & $0.00 \%$ & $0.00 \%$ & $2.78 \%$ \\
PPI & $0.00 \%$ & $2.63 \%$ & $0.00 \%$ & $0.00 \%$ & $0.00 \%$ & $0.00 \%$ & $0.00 \%$ \\
RSA & $0.00 \%$ & $0.00 \%$ & $0.00 \%$ & $0.00 \%$ & $0.00 \%$ & $0.97 \%$ & $0.00 \%$ \\
\hline
\end{tabular}

Continued on next page 
Table 10: Relation between Jumps and Scheduled Announcements Disaggregated by Announcement Items (Non-Crisis and Crisis Subsample) Continued from previous page

\begin{tabular}{ccccccc}
\hline DOTM & OTM & ATM & ATM & OTM & DOTM & Futures \\
Puts & Puts & Puts & Calls & Calls & Calls & \\
\hline Panel B: $P($ News $\mid$ Jump $)$ & over the & Crisis Subsample &
\end{tabular}

\section{Short-Term Options}

$\begin{array}{lccccccc}\text { NFP } & 9.29 \% & 15.49 \% & 6.84 \% & 10.10 \% & 9.80 \% & 6.04 \% & 12.73 \% \\ \text { CCI } & 0.00 \% & 0.00 \% & 1.71 \% & 3.03 \% & 0.00 \% & 0.55 \% & 0.00 \% \\ \text { CPI } & 2.14 \% & 1.41 \% & 2.56 \% & 2.02 \% & 7.84 \% & 0.55 \% & 0.00 \% \\ \text { DGO } & 2.14 \% & 1.41 \% & 3.42 \% & 4.04 \% & 0.00 \% & 2.75 \% & 0.00 \% \\ \text { FOMC } & 1.43 \% & 2.82 \% & 2.56 \% & 2.02 \% & 5.88 \% & 1.10 \% & 7.27 \% \\ \text { GDP } & 1.43 \% & 4.23 \% & 1.71 \% & 4.04 \% & 5.88 \% & 4.40 \% & 1.82 \% \\ \text { IJC } & 12.86 \% & 2.82 \% & 11.11 \% & 7.07 \% & 13.73 \% & 12.09 \% & 0.00 \% \\ \text { LI } & 0.71 \% & 0.00 \% & 0.85 \% & 1.01 \% & 0.00 \% & 0.55 \% & 0.00 \% \\ \text { NHS } & 0.71 \% & 1.41 \% & 0.00 \% & 1.01 \% & 0.00 \% & 1.10 \% & 0.00 \% \\ \text { PPI } & 2.86 \% & 1.41 \% & 0.00 \% & 1.01 \% & 0.00 \% & 1.10 \% & 0.00 \% \\ \text { RSA } & 2.14 \% & 1.41 \% & 0.00 \% & 0.00 \% & 5.88 \% & 1.10 \% & 0.00 \%\end{array}$

Medium-Term Options

$\begin{array}{lccccccc}\text { NFP } & 9.35 \% & 9.46 \% & 7.14 \% & 11.54 \% & 13.11 \% & 2.42 \% & 15.09 \% \\ \text { CCI } & 0.00 \% & 1.35 \% & 1.79 \% & 1.92 \% & 0.00 \% & 0.61 \% & 0.00 \% \\ \text { CPI } & 0.93 \% & 0.00 \% & 0.89 \% & 0.00 \% & 0.00 \% & 2.42 \% & 0.00 \% \\ \text { DGO } & 0.00 \% & 2.70 \% & 0.89 \% & 0.00 \% & 6.56 \% & 1.82 \% & 0.00 \% \\ \text { FOMC } & 2.80 \% & 4.05 \% & 2.68 \% & 3.85 \% & 3.28 \% & 0.61 \% & 7.55 \% \\ \text { GDP } & 0.00 \% & 2.70 \% & 0.00 \% & 1.92 \% & 1.64 \% & 0.61 \% & 1.89 \% \\ \text { IJC } & 5.61 \% & 14.86 \% & 1.79 \% & 7.69 \% & 8.20 \% & 9.70 \% & 0.00 \% \\ \text { LI } & 0.93 \% & 1.35 \% & 0.00 \% & 0.00 \% & 0.00 \% & 1.21 \% & 0.00 \% \\ \text { NHS } & 0.00 \% & 0.00 \% & 0.89 \% & 1.92 \% & 1.64 \% & 0.61 \% & 0.00 \% \\ \text { PPI } & 0.93 \% & 1.35 \% & 0.89 \% & 0.00 \% & 0.00 \% & 1.21 \% & 0.00 \% \\ \text { RSA } & 0.93 \% & 2.70 \% & 0.00 \% & 1.92 \% & 0.00 \% & 0.61 \% & 0.00 \%\end{array}$

Long-Term Options

\begin{tabular}{lccccccc} 
NFP & $5.61 \%$ & $8.82 \%$ & $4.90 \%$ & $11.43 \%$ & $9.46 \%$ & $3.11 \%$ & $12.00 \%$ \\
CCI & $0.00 \%$ & $1.47 \%$ & $1.96 \%$ & $0.00 \%$ & $1.35 \%$ & $0.00 \%$ & $0.00 \%$ \\
CPI & $1.87 \%$ & $0.00 \%$ & $0.98 \%$ & $0.00 \%$ & $6.76 \%$ & $1.55 \%$ & $0.00 \%$ \\
DGO & $3.74 \%$ & $0.00 \%$ & $0.98 \%$ & $0.00 \%$ & $1.35 \%$ & $2.07 \%$ & $0.00 \%$ \\
FOMC & $0.94 \%$ & $2.94 \%$ & $2.94 \%$ & $2.86 \%$ & $2.70 \%$ & $0.52 \%$ & $8.00 \%$ \\
GDP & $1.87 \%$ & $1.47 \%$ & $0.00 \%$ & $2.86 \%$ & $5.41 \%$ & $2.07 \%$ & $2.00 \%$ \\
IJC & $7.48 \%$ & $8.82 \%$ & $0.98 \%$ & $5.71 \%$ & $10.82 \%$ & $5.70 \%$ & $0.00 \%$ \\
LI & $1.87 \%$ & $0.00 \%$ & $0.00 \%$ & $0.00 \%$ & $0.00 \%$ & $1.03 \%$ & $0.00 \%$ \\
NHS & $0.00 \%$ & $1.47 \%$ & $0.98 \%$ & $0.00 \%$ & $0.00 \%$ & $0.52 \%$ & $0.00 \%$ \\
PPI & $0.94 \%$ & $0.00 \%$ & $0.00 \%$ & $0.00 \%$ & $1.35 \%$ & $2.07 \%$ & $0.00 \%$ \\
RSA & $0.94 \%$ & $1.47 \%$ & $0.00 \%$ & $11.43 \%$ & $0.00 \%$ & $3.11 \%$ & $0.00 \%$ \\
\hline
\end{tabular}

Continued on next page 
Table 10: Relation between Jumps and Scheduled Announcements Disaggregated by Announcement Items (Non-Crisis and Crisis Subsample) Continued from previous page

\begin{tabular}{ccccccl}
\hline DOTM & OTM & ATM & ATM & OTM & DOTM & Futures \\
Puts & Puts & Puts & Calls & Calls & Calls & \\
\hline
\end{tabular}

Panel C: $P($ Jump $\mid$ News $)$ over the Non-Crisis Subsample

\section{Short-Term Options}

$\begin{array}{lccccccc}\text { NFP } & 6.45 \% & 12.90 \% & 9.68 \% & 6.45 \% & 6.45 \% & 6.45 \% & 12.90 \% \\ \text { CCI } & 3.33 \% & 0.00 \% & 3.33 \% & 0.00 \% & 0.00 \% & 10.00 \% & 0.00 \% \\ \text { CPI } & 12.90 \% & 9.68 \% & 6.45 \% & 16.13 \% & 6.45 \% & 3.23 \% & 0.00 \% \\ \text { DGO } & 9.68 \% & 0.00 \% & 3.23 \% & 0.00 \% & 6.45 \% & 3.23 \% & 0.00 \% \\ \text { FOMC } & 0.00 \% & 0.00 \% & 5.00 \% & 5.00 \% & 0.00 \% & 0.00 \% & 25.00 \% \\ \text { GDP } & 12.90 \% & 0.00 \% & 3.23 \% & 9.68 \% & 3.23 \% & 0.00 \% & 0.00 \% \\ \text { IJC } & 3.73 \% & 5.97 \% & 0.00 \% & 8.96 \% & 6.72 \% & 2.99 \% & 0.75 \% \\ \text { LI } & 0.00 \% & 0.00 \% & 0.00 \% & 0.00 \% & 0.00 \% & 0.00 \% & 0.00 \% \\ \text { NHS } & 0.00 \% & 0.00 \% & 0.00 \% & 3.23 \% & 0.00 \% & 0.00 \% & 3.23 \% \\ \text { PPI } & 3.23 \% & 19.35 \% & 0.00 \% & 6.45 \% & 6.45 \% & 9.68 \% & 0.00 \% \\ \text { RSA } & 0.00 \% & 16.13 \% & 0.00 \% & 6.45 \% & 3.23 \% & 3.23 \% & 0.00 \%\end{array}$

\section{Medium-Term Options}

$\begin{array}{lccccccc}\text { NFP } & 12.90 \% & 12.90 \% & 3.23 \% & 0.00 \% & 22.58 \% & 6.45 \% & 16.13 \% \\ \text { CCI } & 0.00 \% & 0.00 \% & 3.33 \% & 3.33 \% & 3.33 \% & 0.00 \% & 0.00 \% \\ \text { CPI } & 3.23 \% & 3.23 \% & 6.45 \% & 3.23 \% & 6.45 \% & 6.45 \% & 0.00 \% \\ \text { DGO } & 3.23 \% & 0.00 \% & 0.00 \% & 0.00 \% & 0.00 \% & 3.23 \% & 0.00 \% \\ \text { FOMC } & 5.00 \% & 5.00 \% & 5.00 \% & 0.00 \% & 0.00 \% & 5.00 \% & 25.00 \% \\ \text { GDP } & 3.23 \% & 9.68 \% & 0.00 \% & 3.23 \% & 3.23 \% & 3.23 \% & 0.00 \% \\ \text { IJC } & 0.75 \% & 1.49 \% & 0.75 \% & 0.75 \% & 2.24 \% & 0.00 \% & 0.75 \% \\ \text { LI } & 0.00 \% & 0.00 \% & 0.00 \% & 0.00 \% & 3.23 \% & 3.23 \% & 0.00 \% \\ \text { NHS } & 0.00 \% & 0.00 \% & 0.00 \% & 3.23 \% & 0.00 \% & 3.23 \% & 3.23 \% \\ \text { PPI } & 6.45 \% & 0.00 \% & 0.00 \% & 0.00 \% & 0.00 \% & 0.00 \% & 0.00 \% \\ \text { RSA } & 0.00 \% & 0.00 \% & 0.00 \% & 0.00 \% & 3.23 \% & 3.23 \% & 0.00 \%\end{array}$

\section{Long-Term Options}

\begin{tabular}{lccccccc} 
NFP & $3.23 \%$ & $3.23 \%$ & $3.23 \%$ & $0.00 \%$ & $6.45 \%$ & $9.68 \%$ & $9.68 \%$ \\
CCI & $10.00 \%$ & $3.33 \%$ & $3.33 \%$ & $0.00 \%$ & $10.00 \%$ & $0.00 \%$ & $6.67 \%$ \\
CPI & $0.00 \%$ & $0.00 \%$ & $3.23 \%$ & $0.00 \%$ & $6.45 \%$ & $0.00 \%$ & $0.00 \%$ \\
DGO & $6.45 \%$ & $0.00 \%$ & $0.00 \%$ & $0.00 \%$ & $0.00 \%$ & $3.23 \%$ & $0.00 \%$ \\
FOMC & $0.00 \%$ & $10.00 \%$ & $10.00 \%$ & $0.00 \%$ & $0.00 \%$ & $15.00 \%$ & $25.00 \%$ \\
GDP & $0.00 \%$ & $0.00 \%$ & $0.00 \%$ & $0.00 \%$ & $0.00 \%$ & $3.23 \%$ & $0.00 \%$ \\
IJC & $0.75 \%$ & $0.00 \%$ & $0.00 \%$ & $0.00 \%$ & $1.49 \%$ & $0.00 \%$ & $0.75 \%$ \\
LI & $3.23 \%$ & $0.00 \%$ & $0.00 \%$ & $0.00 \%$ & $0.00 \%$ & $0.00 \%$ & $0.00 \%$ \\
NHS & $6.45 \%$ & $0.00 \%$ & $6.45 \%$ & $3.23 \%$ & $0.00 \%$ & $0.00 \%$ & $3.23 \%$ \\
PPI & $0.00 \%$ & $3.23 \%$ & $0.00 \%$ & $0.00 \%$ & $0.00 \%$ & $0.00 \%$ & $0.00 \%$ \\
RSA & $0.00 \%$ & $0.00 \%$ & $0.00 \%$ & $0.00 \%$ & $0.00 \%$ & $3.23 \%$ & $0.00 \%$ \\
\hline
\end{tabular}


Table 10: Relation between Jumps and Scheduled Announcements Disaggregated by Announcement Items (Non-Crisis and Crisis Subsample)

Continued from previous page

\begin{tabular}{|c|c|c|c|c|c|c|c|}
\hline & $\begin{array}{c}\text { DOTM } \\
\text { Puts }\end{array}$ & $\begin{array}{l}\text { OTM } \\
\text { Puts }\end{array}$ & $\begin{array}{l}\text { ATM } \\
\text { Puts }\end{array}$ & $\begin{array}{l}\text { ATM } \\
\text { Calls }\end{array}$ & $\begin{array}{l}\text { OTM } \\
\text { Calls }\end{array}$ & $\begin{array}{c}\text { DOTM } \\
\text { Calls }\end{array}$ & Futures \\
\hline \multicolumn{8}{|c|}{ Panel D: P(Jump $\mid$ News $)$ over the Crisis Subsample } \\
\hline \multicolumn{8}{|c|}{ Short-Term Options } \\
\hline NFP & $31.71 \%$ & $26.83 \%$ & $19.51 \%$ & $24.39 \%$ & $12.20 \%$ & $26.83 \%$ & $17.07 \%$ \\
\hline CCI & $0.00 \%$ & $0.00 \%$ & $4.88 \%$ & $7.32 \%$ & $0.00 \%$ & $2.44 \%$ & $0.00 \%$ \\
\hline CPI & $7.32 \%$ & $2.44 \%$ & $7.32 \%$ & $4.88 \%$ & $9.76 \%$ & $2.44 \%$ & $0.00 \%$ \\
\hline DGO & $7.32 \%$ & $2.44 \%$ & $9.76 \%$ & $9.76 \%$ & $0.00 \%$ & $12.20 \%$ & $0.00 \%$ \\
\hline FOMC & $6.67 \%$ & $6.67 \%$ & $10.00 \%$ & $6.67 \%$ & $10.00 \%$ & $6.67 \%$ & $13.33 \%$ \\
\hline GDP & $4.88 \%$ & $7.32 \%$ & $4.88 \%$ & $9.76 \%$ & $7.32 \%$ & $19.51 \%$ & $2.44 \%$ \\
\hline IJC & $10.06 \%$ & $1.12 \%$ & $7.26 \%$ & $3.91 \%$ & $3.91 \%$ & $12.29 \%$ & $0.00 \%$ \\
\hline LI & $2.44 \%$ & $0.00 \%$ & $2.44 \%$ & $2.44 \%$ & $0.00 \%$ & $2.44 \%$ & $0.00 \%$ \\
\hline NHS & $2.44 \%$ & $2.44 \%$ & $0.00 \%$ & $2.44 \%$ & $0.00 \%$ & $4.88 \%$ & $0.00 \%$ \\
\hline PPI & $9.76 \%$ & $2.44 \%$ & $0.00 \%$ & $2.44 \%$ & $0.00 \%$ & $4.88 \%$ & $0.00 \%$ \\
\hline RSA & $7.32 \%$ & $2.44 \%$ & $0.00 \%$ & $0.00 \%$ & $7.32 \%$ & $4.88 \%$ & $0.00 \%$ \\
\hline
\end{tabular}

Medium-Term Options

$\begin{array}{lccccccc}\text { NFP } & 24.39 \% & 17.07 \% & 19.51 \% & 14.63 \% & 19.51 \% & 9.76 \% & 19.51 \% \\ \text { CCI } & 0.00 \% & 2.44 \% & 4.88 \% & 2.44 \% & 0.00 \% & 2.44 \% & 0.00 \% \\ \text { CPI } & 2.44 \% & 0.00 \% & 2.44 \% & 0.00 \% & 0.00 \% & 9.76 \% & 0.00 \% \\ \text { DGO } & 0.00 \% & 4.88 \% & 2.44 \% & 0.00 \% & 9.76 \% & 7.32 \% & 0.00 \% \\ \text { FOMC } & 10.00 \% & 10.00 \% & 10.00 \% & 6.67 \% & 6.67 \% & 3.33 \% & 13.33 \% \\ \text { GDP } & 0.00 \% & 4.88 \% & 0.00 \% & 2.44 \% & 2.44 \% & 2.44 \% & 2.44 \% \\ \text { IJC } & 3.35 \% & 6.15 \% & 1.12 \% & 2.23 \% & 2.79 \% & 8.94 \% & 0.00 \% \\ \text { LI } & 2.44 \% & 2.44 \% & 0.00 \% & 0.00 \% & 0.00 \% & 4.88 \% & 0.00 \% \\ \text { NHS } & 0.00 \% & 0.00 \% & 2.44 \% & 2.44 \% & 2.44 \% & 2.44 \% & 0.00 \% \\ \text { PPI } & 2.44 \% & 2.44 \% & 2.44 \% & 0.00 \% & 0.00 \% & 4.88 \% & 0.00 \% \\ \text { RSA } & 2.44 \% & 4.88 \% & 0.00 \% & 2.44 \% & 0.00 \% & 2.44 \% & 0.00 \%\end{array}$

Long-Term Options

$\begin{array}{lccccccc}\text { NFP } & 14.63 \% & 14.63 \% & 12.20 \% & 9.76 \% & 17.07 \% & 14.63 \% & 14.63 \% \\ \text { CCI } & 0.00 \% & 2.44 \% & 4.88 \% & 0.00 \% & 2.44 \% & 0.00 \% & 0.00 \% \\ \text { CPI } & 4.88 \% & 0.00 \% & 2.44 \% & 0.00 \% & 12.20 \% & 7.32 \% & 0.00 \% \\ \text { DGO } & 9.76 \% & 0.00 \% & 2.44 \% & 0.00 \% & 2.44 \% & 9.76 \% & 0.00 \% \\ \text { FOMC } & 3.33 \% & 6.67 \% & 10.00 \% & 3.33 \% & 6.67 \% & 3.33 \% & 13.33 \% \\ \text { GDP } & 4.88 \% & 2.44 \% & 0.00 \% & 2.44 \% & 9.76 \% & 9.76 \% & 2.44 \% \\ \text { IJC } & 4.47 \% & 3.35 \% & 0.56 \% & 1.12 \% & 4.47 \% & 6.15 \% & 0.00 \% \\ \text { LI } & 4.88 \% & 0.00 \% & 0.00 \% & 0.00 \% & 0.00 \% & 4.88 \% & 0.00 \% \\ \text { NHS } & 0.00 \% & 2.44 \% & 2.44 \% & 0.00 \% & 0.00 \% & 2.44 \% & 0.00 \% \\ \text { PPI } & 2.44 \% & 0.00 \% & 0.00 \% & 0.00 \% & 2.44 \% & 9.76 \% & 0.00 \% \\ \text { RSA } & 2.44 \% & 2.44 \% & 0.00 \% & 9.76 \% & 0.00 \% & 14.63 \% & 0.00 \%\end{array}$

Entries report summary statistics on the relation between detected jumps and macroeconomic news announcements disaggregated by news items for all investigated moneyness and maturity categories over the non-crisis subsample (Panels A and C) and the crisis subsample (Panels B and D). The probability of a jump to be related to a specific news announcement $P($ News $\mid$ Jump) (Panels A and B) and the probability of a specific news announcement leading to a jump $P($ Jump $\mid$ News) (Panels C and D) are reported. Jumps have been detected using the Lee and Mykland (2008) jump detection methodology based on a significance level $\alpha=0.1 \%$. Jumps are defined to be related to a news announcement if they occurred within \pm 10 minutes of an announcement. The sample period is 1 January 2005 to 31 July 2007 for the non-crisis subsample and 1 August 2007 to 31 December 2010 for the crisis subsample. 
Table 11: Information Shocks, Volume and Illiquidity as jump determinants (Crisis Subsample)

\begin{tabular}{lccc}
\hline & Short-Term & Medium-Term & Long-Term \\
\hline & & & \\
$c$ & $-3.2964^{* * *}$ & $-3.4553^{* * *}$ & $-3.9756^{* * *}$ \\
sBA $_{t-1}$ & $0.0706^{*}$ & $0.132^{* * *}$ & $0.1571^{* * *}$ \\
BidSize $_{t-1}$ & -0.0017 & -0.0019 & -0.0005 \\
AskSize $_{t-1}$ & -0.0005 & -0.0032 & $-0.0089^{* *}$ \\
Volume $_{t-1, t}$ & -0.0053 & $0.0035^{*}$ & 0.0017 \\
NFP $_{t}$ & $0.5986^{* * *}$ & 0.2969 & -0.0498 \\
CCI & -1.7225 & -10.3653 & - \\
CPI & -0.1908 & -1.0597 & 0.0343 \\
DGO $_{t}$ & -0.1901 & -0.9037 & -1.4842 \\
$F O M C_{t}$ & - & - & - \\
GDP & 0.0403 & -1.5392 & $0.6491^{* * *}$ \\
IJC $_{t}$ & -0.0839 & -0.1693 & 0.036 \\
II $_{t}$ & -0.0886 & -0.3529 & - \\
$N H S_{t}$ & - & -4.8425 & - \\
PPI $_{t}$ & -0.3548 & -1.9787 & - \\
RSA $_{t}$ & -0.3868 & -0.8008 & 0.485 \\
\hline
\end{tabular}

Entries report the estimation results for the logistic regression model in equation (11) over the crisis subsample. The estimation is performed separately for short, medium, and long-term options on a sample pooled across all delta categories. Only news-related observations are considered. The estimation is performed via Maximum Likelihood and $* * *, * *$, or * report statistical significance on a $1 \%, 5 \%$, or $10 \%$ significance level. The sample period is $1 / 8 / 2007$ to $31 / 12 / 2010$. 
Table 12: Relation between Jumps and Unscheduled News Announcements

\begin{tabular}{lccccccc}
\hline & $\begin{array}{c}\text { DOTM } \\
\text { Puts }\end{array}$ & $\begin{array}{c}\text { OTM } \\
\text { Puts }\end{array}$ & $\begin{array}{c}\text { ATM } \\
\text { Puts }\end{array}$ & $\begin{array}{c}\text { ATM } \\
\text { Calls }\end{array}$ & $\begin{array}{c}\text { OTM } \\
\text { Calls }\end{array}$ & $\begin{array}{c}\text { DOTM } \\
\text { Calls }\end{array}$ & Futures \\
\hline $\begin{array}{l}\text { Short-Term Options } \\
\text { \# Jump Days equal to }\end{array}$ & & & & & & & \\
$\begin{array}{l}\text { Unsched. News Day } \\
\text { \% Jump Days equal to } \\
\text { \% Unsched. News Day }\end{array}$ & 5 & 4 & 3 & 2 & 0 & 10 & 3 \\
$\begin{array}{l}\text { Medium-Term Options } \\
\text { \# Jump Days equal to }\end{array}$ & $2.16 \%$ & $2.99 \%$ & $2.34 \%$ & $1.24 \%$ & $0.00 \%$ & $4.07 \%$ & $4.17 \%$ \\
$\begin{array}{l}\text { Unsched. News Day } \\
\text { \% Jump Days equal to }\end{array}$ & 3 & 4 & 7 & 3 & 3 & 7 & 3 \\
$\begin{array}{l}\text { Unsched. News Day } \\
\text { Long-Term Options }\end{array}$ & $1.66 \%$ & $4.00 \%$ & $6.31 \%$ & $3.75 \%$ & $2.40 \%$ & $3.21 \%$ & $4.29 \%$ \\
$\begin{array}{l}\text { \# Jump Days equal to } \\
\text { Unsched. News Day } \\
\% \text { Jump Days equal to }\end{array}$ & 4 & 4 & 7 & 1 & 1 & 9 & 3 \\
\begin{tabular}{l} 
Unsched. News Day \\
\hline
\end{tabular}
\end{tabular}

Entries report summary statistics on the relation between detected jumps and unscheduled news announcements for all investigated moneyness and maturity categories (Panels A to C). The number of jump days that are equal to a day on which unscheduled news has been released and this number as a fraction of all jump days is reported. Jumps have been detected using the Lee and Mykland (2008) jump detection methodology based on a significance level $\alpha=0.1 \%$. The sample period is 1 January 2005 to 31 December 2010. 


\section{Figures}

Figure 1: Volatility Signature Plots of Short-Term Options Returns
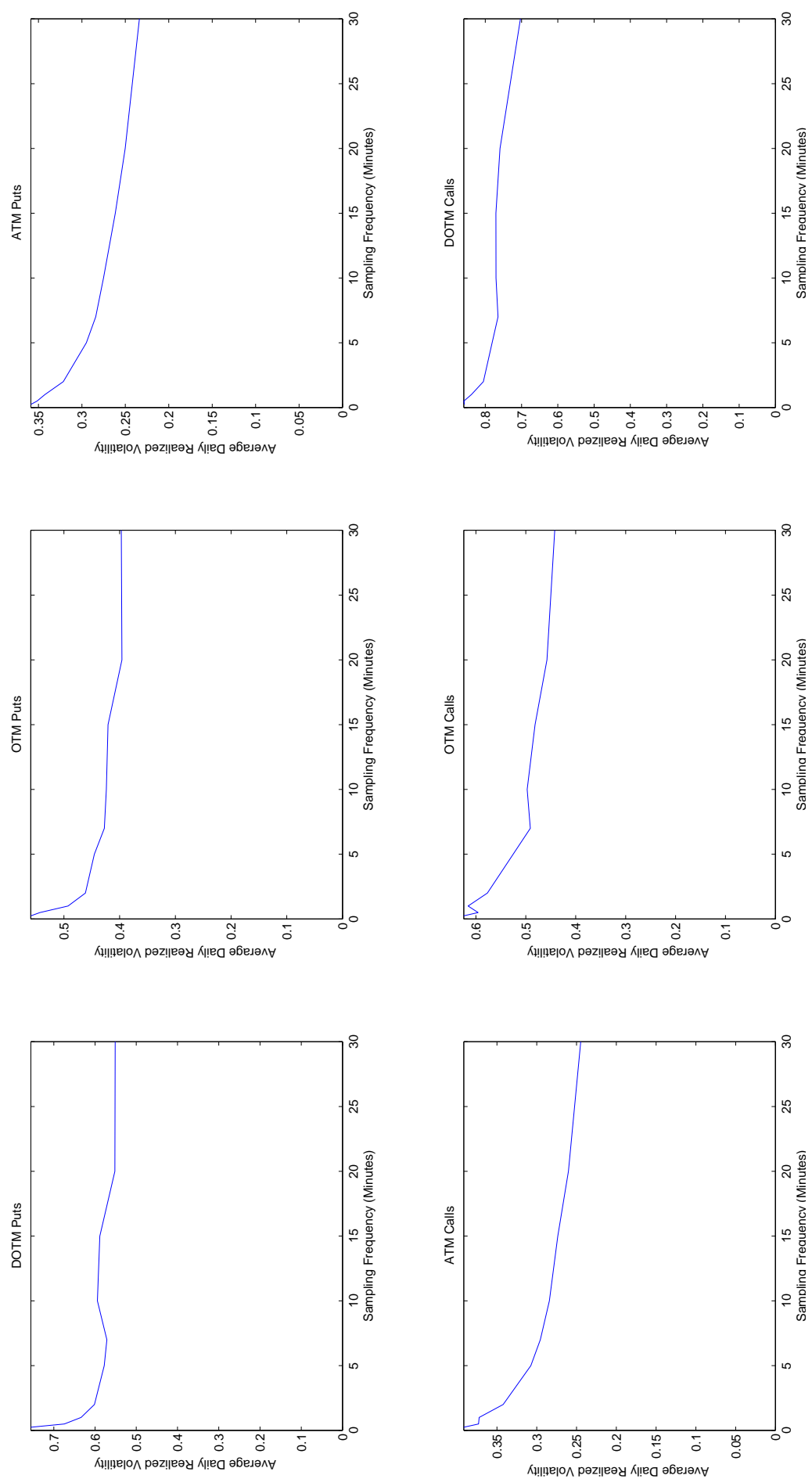

The figure depicts the average daily realized volatility of option returns as a function of the sampling frequency for short-term options of different delta categories. We consider the following delta categories (defined in Table 1) for calls and puts: deep out-of-the-money (DOTM), out-of-the-money (OTM) and at-the-money (ATM). 
Figure 2: Distribution of Co-Jumps
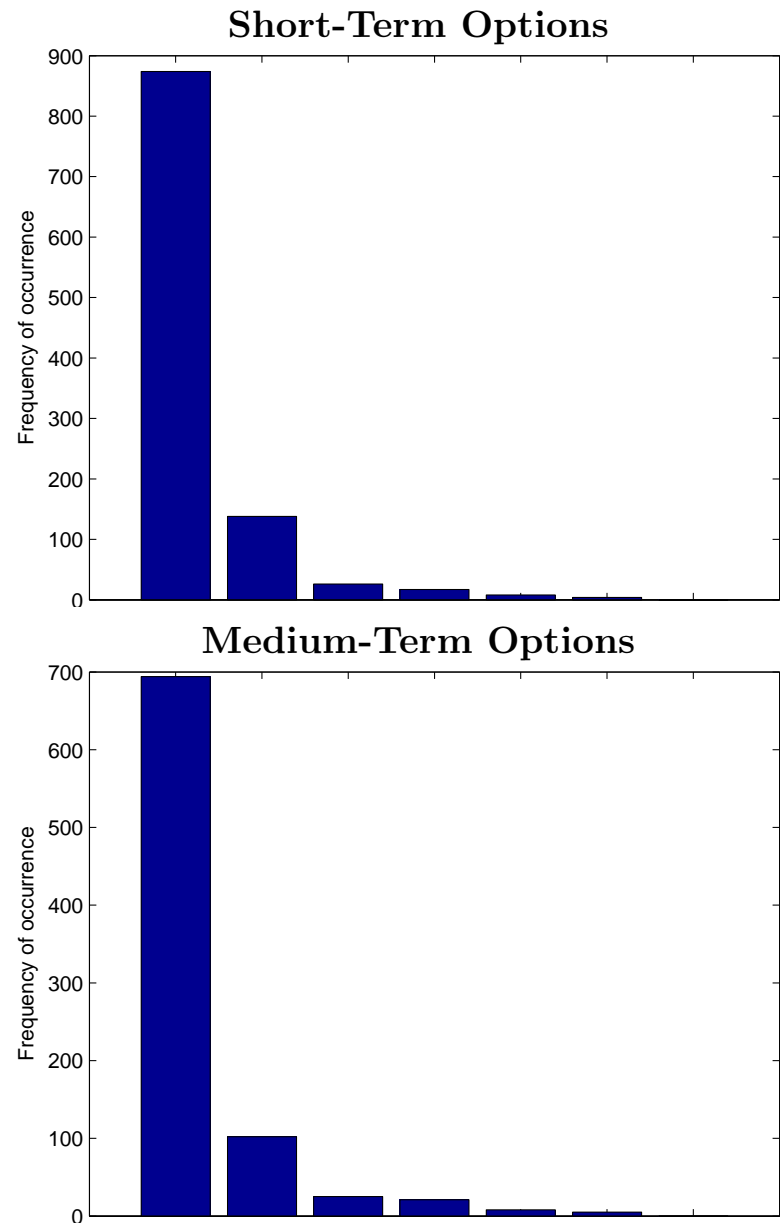

Long-Term Options

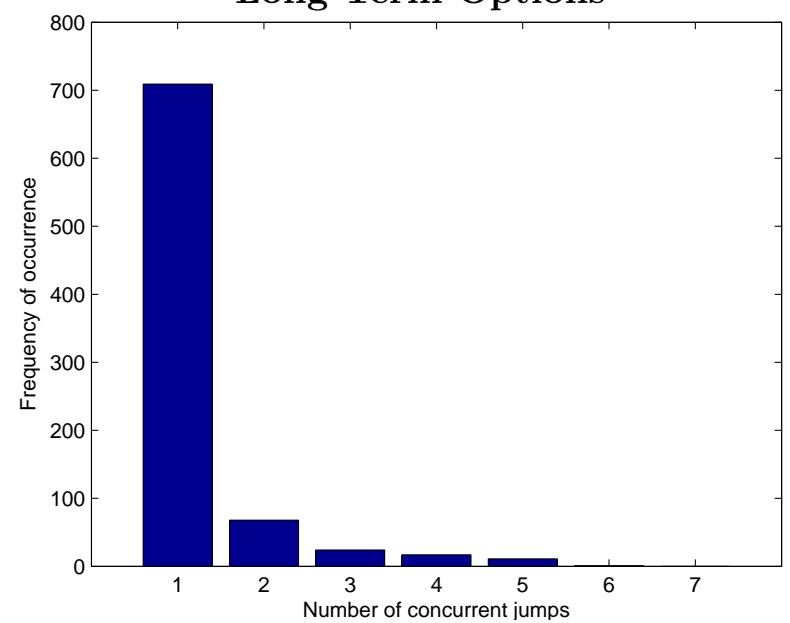

The figure illustrates the distribution of co-jump events for short, medium, and long-term options, separately. Co-jump events are defined by the number of concurrent jumps across different delta levels and the underlying asset. The event of only one concurrent jump corresponds to an idiosyncratic jump in only one of the delta categories or the underlying asset. The frequency of occurrence is reported for each possible co-jump event. 
Figure 3: Composition of Co-Jumps

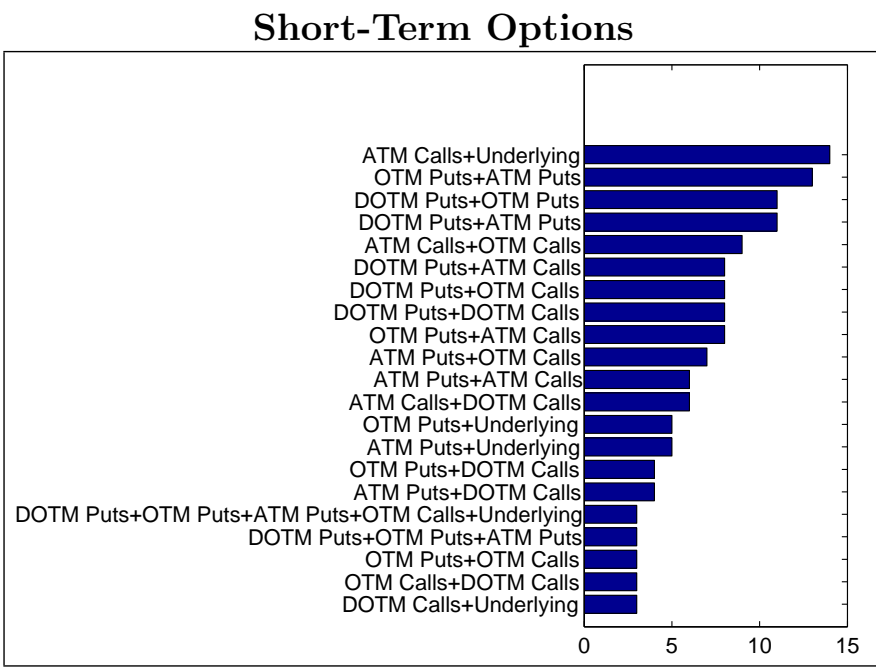

Medium-Term Options

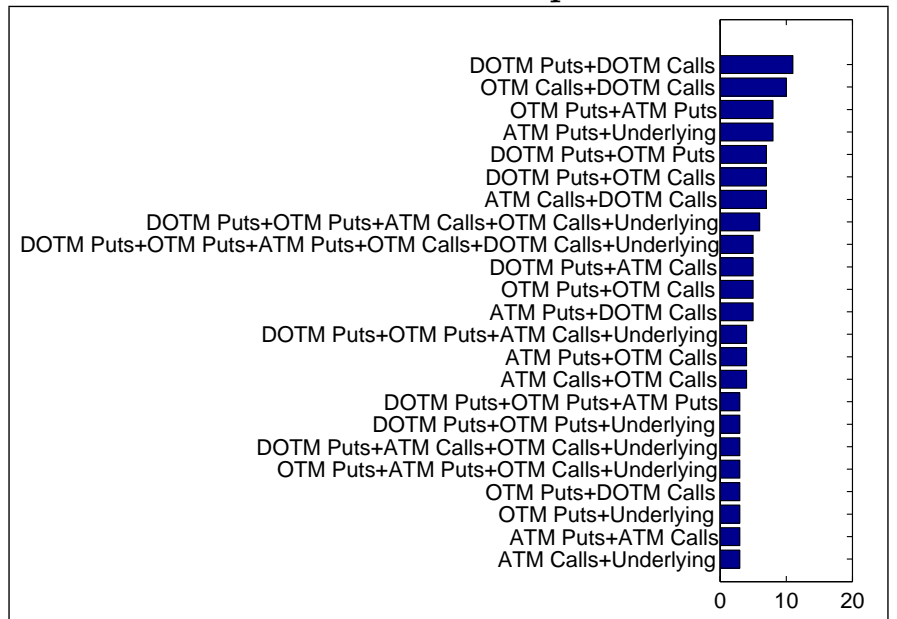

Long-Term Options

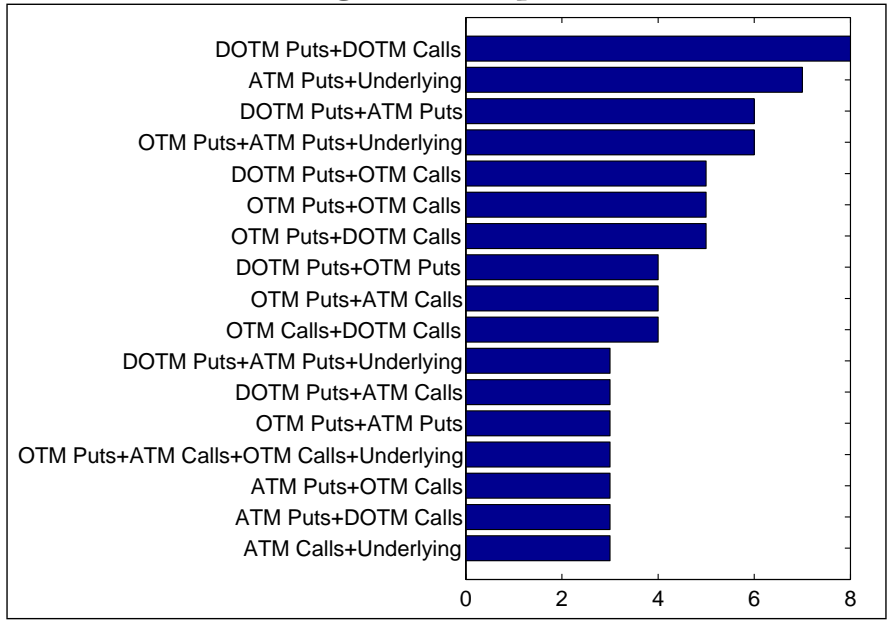

The figure illustrates the composition of the most frequent co-jump events for short-term, medium-term, and long-term options. The composition of a co-jump event is characterized by the delta categories of the options and/or the underlying asset that simultaneously exhibit a jump. 
Figure 4: Option Bid-ask Spreads around Jumps
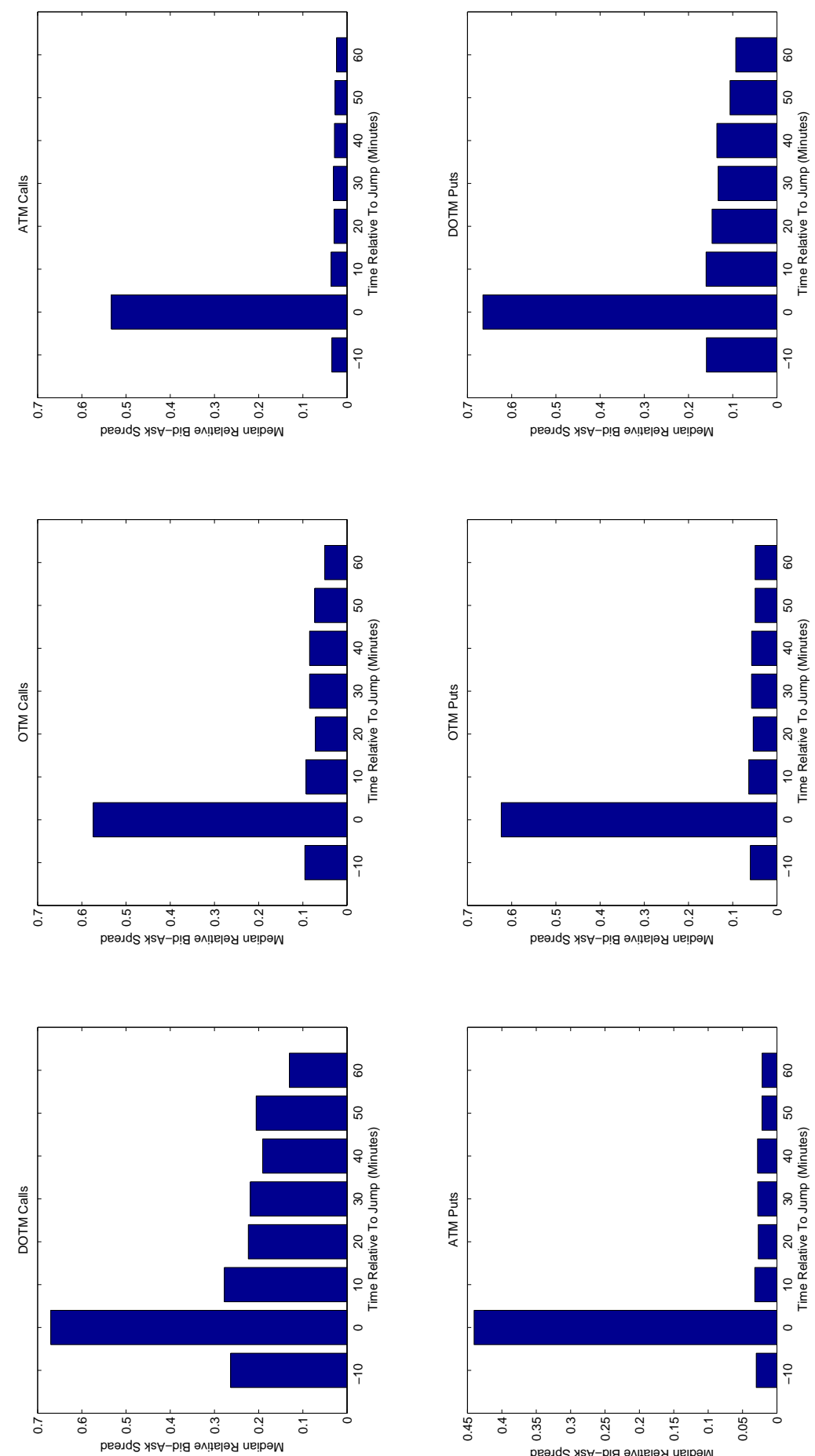

The figure illustrates the median relative option bid-ask spreads for a number of time subintervals around the news related jumps (10 minutes before the jump up to 60 minutes after the jump) across the various moneyness levels for the case of the short maturity options. We consider deep out-of-themoney (DOTM), out-of-the-money (OTM) and at-the-money (ATM) calls and puts (defined in Table 1). The jump time corresponds to point zero in the graph. 
Figure 5: Distribution of Option Trading Volume Before Short-Term News-Related Option Price Jumps
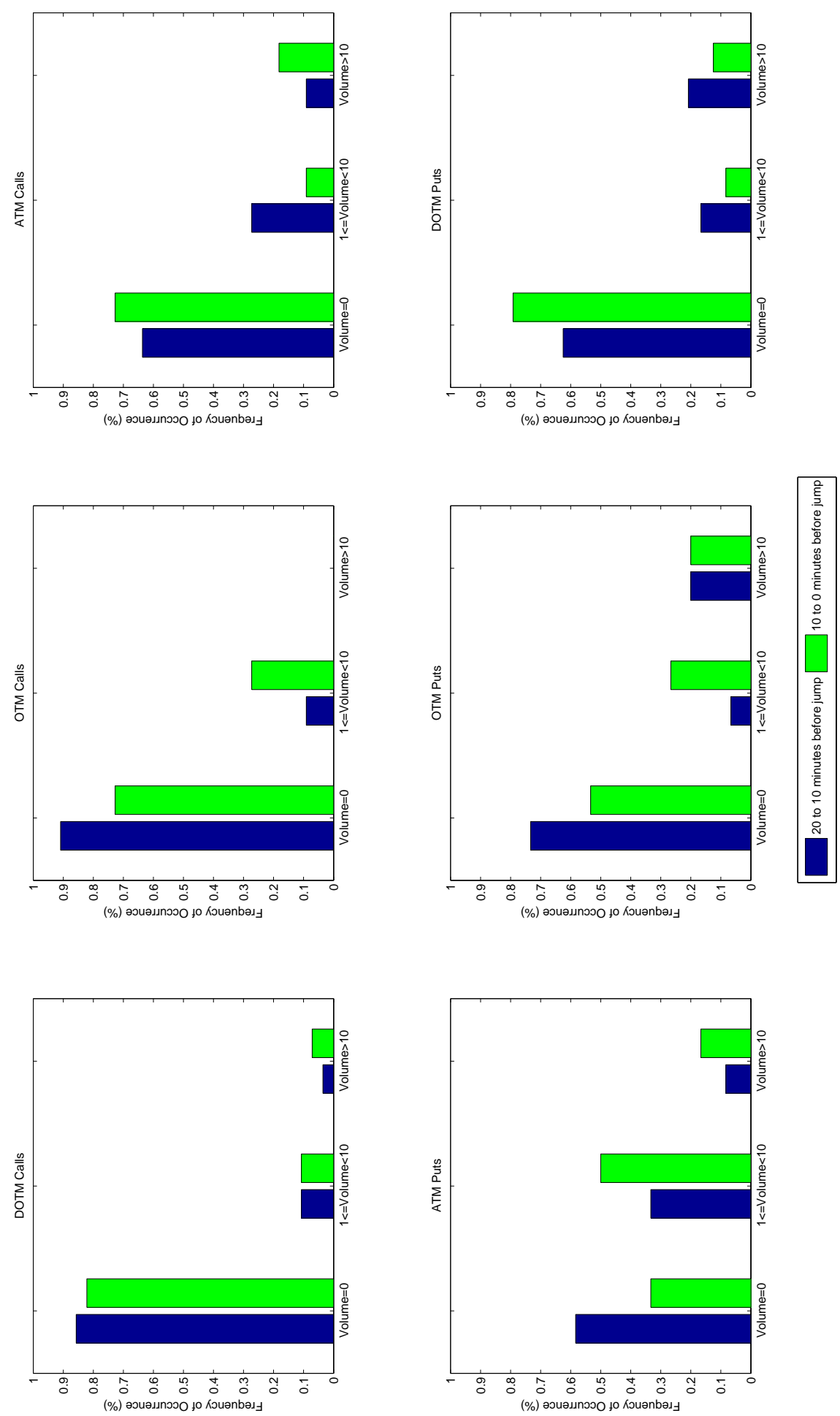

The figure illustrates the frequency (in \%) distribution in the two 10-minutes subintervals before newsrelated short-term option price jumps. The frequency distribution is constructed for option trading volume buckets of zero, 10 or more than 10 contracts. We consider deep out-of-the-money (DOTM), out-of-the-money (OTM) and at-the-money (ATM) calls and puts, (defined in Table 1). 
Figure 6: Dynamics of Short-Term Options Bid-Ask Spreads
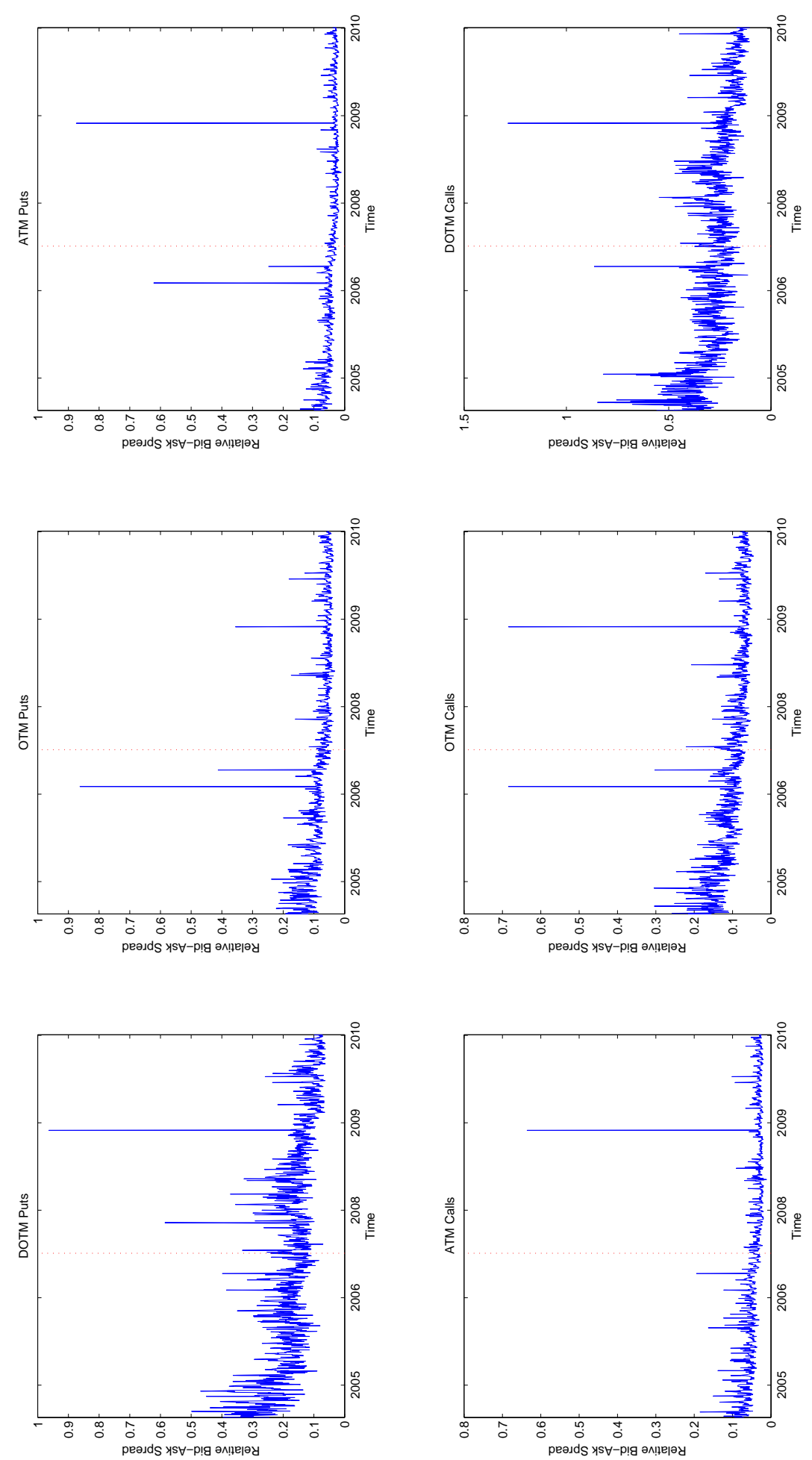

The figure illustrates the time evolution of the relative bid-ask spread of short-term options of different delta categories over the non-crisis and crisis subsample. We consider deep out-of-the-money (DOTM), out-of-the-money (OTM) and at-the-money (ATM) calls and puts, (defined in Table 1). The daily average relative-bid ask spread is depicted. The dashed line illustrates the non-crisis/crisis split point. 